\title{
A Common Structure in PBW Bases of the Nilpotent Subalgebra of $U_{q}(\mathfrak{g})$ and Quantized Algebra of Functions ${ }^{\star}$
}

\author{
Atsuo KUNIBA ${ }^{\dagger}$, Masato OKADO ${ }^{\ddagger}$ and Yasuhiko YAMADA $\S$ \\ † Institute of Physics, Graduate School of Arts and Sciences, University of Tokyo, \\ Komaba, Tokyo 153-8902, Japan \\ E-mail:atsuo@gokutan.c.u-tokyo.ac.jp \\ $¥$ Department of Mathematical Science, Graduate School of Engineering Science, \\ Osaka University, Toyonaka, Osaka 560-8531, Japan \\ E-mail: okado@sigmath.es.osaka-u.ac.jp \\ $\S$ Department of Mathematics, Faculty of Science, Kobe University, Hyogo 657-8501, Japan \\ E-mail: yamaday@math.kobe-u.ac.jp
}

Received March 19, 2013, in final form July 10, 2013; Published online July 19, 2013 http://dx.doi.org/10.3842/SIGMA.2013.049

\begin{abstract}
For a finite-dimensional simple Lie algebra $\mathfrak{g}$, let $U_{q}^{+}(\mathfrak{g})$ be the positive part of the quantized universal enveloping algebra, and $A_{q}(\mathfrak{g})$ be the quantized algebra of functions. We show that the transition matrix of the PBW bases of $U_{q}^{+}(\mathfrak{g})$ coincides with the intertwiner between the irreducible $A_{q}(\mathfrak{g})$-modules labeled by two different reduced expressions of the longest element of the Weyl group of $\mathfrak{g}$. This generalizes the earlier result by Sergeev on $A_{2}$ related to the tetrahedron equation and endows a new representation theoretical interpretation with the recent solution to the $3 \mathrm{D}$ reflection equation for $C_{2}$. Our proof is based on a realization of $U_{q}^{+}(\mathfrak{g})$ in a quotient ring of $A_{q}(\mathfrak{g})$.
\end{abstract}

Key words: quantized enveloping algebra; PBW bases; quantized algebra of functions; tetrahedron equation

2010 Mathematics Subject Classification: 17B37; 20G42; 81R50; 17B80

Dedicated to Professors Anatol N. Kirillov and Tetsuji Miwa who taught us the joy of doing mathematics.

\section{Introduction}

Let $\mathfrak{g}$ be a finite-dimensional simple Lie algebra and $U_{q}(\mathfrak{g})$ be the Drinfeld--Jimbo quantized enveloping algebra. $U_{q}(\mathfrak{g})$ has the subalgebra $U_{q}^{+}(\mathfrak{g})$ generated by the Chevalley generators $e_{1}, \ldots, e_{n}(n=$ rank $\mathfrak{g})$ corresponding to the simple roots. Denote by $W=\left\langle s_{1}, \ldots, s_{n}\right\rangle$ the Weyl group of $\mathfrak{g}$ generated by the simple reflections $s_{1}, \ldots, s_{n}$. It is well known (see for example [15]) that for each reduced expression $w_{0}=s_{i_{1}} \cdots s_{i_{l}}$ of the longest element $w_{0} \in W$, one can associate the Poincaré-Birkhoff-Witt (PBW) basis of $U_{q}^{+}(\mathfrak{g})$ having the form

$$
E_{\mathbf{i}}^{A}=e_{\beta_{1}}^{\left(a_{1}\right)} e_{\beta_{2}}^{\left(a_{2}\right)} \cdots e_{\beta_{l}}^{\left(a_{l}\right)}, \quad A=\left(a_{1}, \ldots, a_{l}\right) \in\left(\mathbb{Z}_{\geq 0}\right)^{l},
$$

where $e_{\beta_{i}}^{\left(a_{i}\right)}$, s are the divided powers of the positive root vectors determined by the choice $\mathbf{i}=$ $\left(i_{1}, \ldots, i_{l}\right)$. See Section 2.2. Let $\left\{E_{\mathbf{j}}^{A} \mid A \in\left(\mathbb{Z}_{\geq 0}\right)^{l}\right\}$ with $\mathbf{j}=\left(j_{1}, \ldots, j_{l}\right)$ be another PBW basis

\footnotetext{
*This paper is a contribution to the Special Issue in honor of Anatol Kirillov and Tetsuji Miwa. The full collection is available at http://www.emis.de/journals/SIGMA/InfiniteAnalysis2013.html
} 
associated with a yet different reduced expression $w_{0}=s_{j_{1}} \cdots s_{j_{l}}$. Following Lusztig [14], one expands a basis in terms of another as

$$
E_{\mathbf{i}}^{A}=\sum_{B \in\left(\mathbb{Z}_{\geq 0}\right)^{l}} \gamma_{B}^{A} E_{\mathbf{j}}^{B}
$$

and obtains the transition coefficient $\gamma_{B}^{A}$ uniquely. We have suppressed its dependence on $\mathbf{i}, \mathbf{j}$ in the notation. Many remarkable properties are known for $\gamma_{B}^{A}$ including the fact $\gamma_{B}^{A} \in \mathbb{Z}[q]$. See [14, Proposition 2.3] for example.

In this paper we show that the transition coefficients $\gamma=\left(\gamma_{B}^{A}\right)$ coincide with the matrix elements of the intertwiner between the irreducible $A_{q}(\mathfrak{g})$-modules labeled by two different reduced expressions of the longest element of the Weyl group of $\mathfrak{g}$. Here $A_{q}(\mathfrak{g})$ denotes the quantized algebra of functions associated with $\mathfrak{g}$. It is a Hopf subalgebra of the dual $U_{q}(\mathfrak{g})^{*}$ which has been studied from a variety of aspects. See [5, 11, 17, 18, 21, 22, 23] for example. Let us briefly recall the most relevant result to the present paper due to Vaksman and Soibelman [21, 22, 23]. To each reduced expression of a (not necessarily longest) element $w=s_{i_{1}} \cdots s_{i_{r}} \in W$, one can associate an irreducible representation $\pi_{\mathbf{i}}$ labeled by $\mathbf{i}=\left(i_{1}, \ldots, i_{r}\right)$ having the form

$$
\pi_{\mathbf{i}}=\pi_{i_{1}} \otimes \cdots \otimes \pi_{i_{r}}: A_{q}(\mathfrak{g}) \rightarrow \operatorname{End}\left(\mathcal{F}_{q_{i_{1}}} \otimes \cdots \otimes \mathcal{F}_{q_{i_{r}}}\right)
$$

where each component $\pi_{i}: A_{q}(\mathfrak{g}) \rightarrow \operatorname{End}\left(\mathcal{F}_{q_{i}}\right)$ is the fundamental representation of $A_{q}(\mathfrak{g})$ on the $q$-oscillator Fock space $\mathcal{F}_{q_{i}}=\bigoplus_{m \geq 0} \mathbb{C}(q)|m\rangle$. See Section 4.1. The two irreducible representations $\pi_{\mathbf{i}}$ and $\pi_{\mathbf{j}}$ with $\mathbf{j}=\left(j_{1}, \ldots, j_{r}\right)$ are isomorphic if $s_{i_{1}} \cdots s_{i_{r}}=s_{j_{1}} \cdots s_{j_{r}} \in W$ are reduced expressions (Theorem 4). Thus one has the intertwiner $\Phi=\Phi_{\mathbf{i}, \mathbf{j}}: \mathcal{F}_{q_{i_{1}}} \otimes \cdots \otimes \mathcal{F}_{q_{i_{r}}} \rightarrow \mathcal{F}_{q_{j_{1}}} \otimes \cdots \otimes \mathcal{F}_{q_{j_{r}}}$ characterized by

$$
\pi_{\mathbf{j}}(g) \circ \Phi=\Phi \circ \pi_{\mathbf{i}}(g) \quad \forall g \in A_{q}(\mathfrak{g})
$$

up to an overall constant. Writing the basis of the Fock space $\mathcal{F}_{q_{i_{1}}} \otimes \cdots \otimes \mathcal{F}_{q_{i_{r}}}$ as $|A\rangle=$ $\left|a_{1}\right\rangle \otimes \cdots \otimes\left|a_{r}\right\rangle$ with $A=\left(a_{1}, \ldots, a_{r}\right) \in\left(\mathbb{Z}_{\geq 0}\right)^{r}$, we define the matrix elements of $\Phi=\left(\Phi_{B}^{A}\right)$ by $\Phi|B\rangle=\sum_{A} \Phi_{B}^{A}|A\rangle$ and the normalization $\Phi_{0, \ldots, 0}^{0, \ldots, 0}=1$. Our main result (Theorem 5) is concerned with the longest element case $r=l$ and is stated for each pair $(\mathbf{i}, \mathbf{j})$ as $\gamma_{B}^{A}=\Phi_{B}^{A}$, i.e.,

$$
\gamma=\Phi
$$

For a convenience we also introduce the "checked" intertwiner $\Phi^{\vee}=\Phi \circ \sigma$, where $\sigma\left(\left|a_{1}\right\rangle \otimes \cdots \otimes\right.$ $\left.\left|a_{l}\right\rangle\right)=\left|a_{l}\right\rangle \otimes \cdots \otimes\left|a_{1}\right\rangle$ is the reversal of the components.

Our work is inspired by recent developments in 3-dimensional (3D) integrable systems related to rank 2 cases. Recall the Zamolodchikov tetrahedron equation [27] and the Isaev-Kulish 3D reflection equation [8]:

$$
\begin{aligned}
& R_{356} R_{246} R_{145} R_{123}=R_{123} R_{145} R_{246} R_{356}, \\
& R_{456} R_{489} K_{3579} R_{269} R_{258} K_{1678} K_{1234}=K_{1234} K_{1678} R_{258} R_{269} K_{3579} R_{489} R_{456} .
\end{aligned}
$$

They are equalities among the linear operators acting on the tensor product of 6 and 9 vector spaces, respectively. The indices specify the components in the tensor product on which the operators $R$ and $K$ act nontrivially. They serve as 3D analogue of the Yang-Baxter and reflection equations postulating certain factorization conditions of straight strings which undergo the scattering $R$ and the reflection $K$ by a boundary plane.

For $\mathfrak{g}=A_{2}$, Kapranov and Voevodsky [10] showed that $R=\Phi^{\vee} \in \operatorname{End}\left(\mathcal{F}_{q}^{\otimes 3}\right)$ provides a solution to the tetrahedron equation (2). Moreover it was discovered by Sergeev [20] that the solution of the tetrahedron equation $R$ in [2] (given also in [10] with misprint) is related with the 
transition matrix as $\gamma=R \circ \sigma$. Thus the equality (1) for $\mathfrak{g}=A_{2}$ is a corollary of their results. Apart from the $A_{2}$ case, it has been shown more recently [13] that $K=\Phi^{\vee}$ for $\mathfrak{g}=C_{2}$ yields the first nontrivial solution to the $3 \mathrm{D}$ reflection equation (3). See also [12] for $\mathfrak{g}=B_{2}$. These results motivated us to investigate the general $\mathfrak{g}$ case and have led to (1). It is our hope that it provides a useful insight into higher-dimensional integrable systems from the representation theory of quantum groups.

The layout of the paper is as follows. In Section 2, we summarize the definitions of $U_{q}(\mathfrak{g})$ and PBW bases. In Section 3, we recall the basic facts on $A_{q}(\mathfrak{g})$ following Kashiwara [11]. A fundamental role is played by the Peter-Weyl type Theorem 1. The relation with the ReshetikhinTakhtadzhyan-Faddeev realization by generators and relations [18] is explained and its concrete forms are quoted for $A_{n}, C_{n}$ and $G_{2}$ [19] which will be of use in later sections. The construction of a certain quotient ring $A_{q}(\mathfrak{g})_{\mathcal{S}}$ of $A_{q}(\mathfrak{g})$ and the special elements $\sigma_{i} \in A_{q}(\mathfrak{g})$ (Definition 1) and $\xi_{i} \in A_{q}(\mathfrak{g})_{\mathcal{S}}(36)$ will play a key role in our proof of (1). In Section 4, we briefly review the representation theory of $A_{q}(\mathfrak{g})$ in $[21,22,23]$ and sketch the intertwiners for the rank 2 cases. Section 5 is devoted to the proof of the main theorem $\gamma=\Phi$. It reduces to the rank 2 cases and is done without recourse to explicit formulae of $\gamma$ or $\Phi$. Our method is to identify their characterizations under the correspondence $e_{i} \mapsto \xi_{i}$. Actually, this map extends to an algebra homomorphism $U_{q}^{+}(\mathfrak{g}) \rightarrow A_{q}(\mathfrak{g})_{\mathcal{S}}$ for general $\mathfrak{g}$ as shown by Yakimov [26]. We give a direct proof of a part of his results in Section 6 .

\section{Quantized enveloping algebra $U_{q}(\mathfrak{g})$}

\subsection{Definition}

In this paper $\mathfrak{g}$ stands for a finite-dimensional simple Lie algebra. Its weight lattice, simple roots, simple coroots, fundamental weights are denoted by $P,\left\{\alpha_{i}\right\}_{i \in I},\left\{h_{i}\right\}_{i \in I},\left\{\varpi_{i}\right\}_{i \in I}$ where $I$ is the index set of the Dynkin diagram of $\mathfrak{g}$. The Cartan matrix $\left(a_{i j}\right)_{i, j \in I}$ is given by $a_{i j}=$ $\left\langle h_{i}, \alpha_{j}\right\rangle=2\left(\alpha_{i}, \alpha_{j}\right) /\left(\alpha_{i}, \alpha_{i}\right)$.

The quantized enveloping algebra $U_{q}(\mathfrak{g})$ is an associative algebra over $\mathbb{Q}(q)$ generated by $\left\{e_{i}, f_{i}, k_{i}^{ \pm 1} \mid i \in I\right\}$ satisfying the relations:

$$
\begin{aligned}
& k_{i} k_{j}=k_{j} k_{i}, \quad k_{i} k_{i}^{-1}=k_{i}^{-1} k_{i}=1, \\
& k_{i} e_{j} k_{i}^{-1}=q_{i}^{\left\langle h_{i}, \alpha_{j}\right\rangle} e_{j}, \quad k_{i} f_{j} k_{i}^{-1}=q_{i}^{-\left\langle h_{i}, \alpha_{j}\right\rangle} f_{j}, \quad\left[e_{i}, f_{j}\right]=\delta_{i j} \frac{k_{i}-k_{i}^{-1}}{q_{i}-q_{i}^{-1}}, \\
& \sum_{r=0}^{1-a_{i j}}(-1)^{r} e_{i}^{(r)} e_{j} e_{i}^{\left(1-a_{i j}-r\right)}=\sum_{r=0}^{1-a_{i j}}(-1)^{r} f_{i}^{(r)} f_{j} f_{i}^{\left(1-a_{i j}-r\right)}=0, \quad i \neq j .
\end{aligned}
$$

Here we use the following notations: $q_{i}=q^{\left(\alpha_{i}, \alpha_{i}\right) / 2},[m]_{i}=\left(q_{i}^{m}-q_{i}^{-m}\right) /\left(q_{i}-q_{i}^{-1}\right),[n]_{i} !=\prod_{m=1}^{n}[m]_{i}$, $e_{i}^{(n)}=e_{i}^{n} /[n]_{i} !, f_{i}^{(n)}=f_{i}^{n} /[n]_{i}$ !. We normalize the simple roots so that $q_{i}=q$ when $\alpha_{i}$ is a short root. $U_{q}(\mathfrak{g})$ is a Hopf algebra. As its comultiplication we adopt the following one

$$
\Delta\left(k_{i}\right)=k_{i} \otimes k_{i}, \quad \Delta\left(e_{i}\right)=e_{i} \otimes 1+k_{i} \otimes e_{i}, \quad \Delta\left(f_{i}\right)=f_{i} \otimes k_{i}^{-1}+1 \otimes f_{i} .
$$

\subsection{PBW basis}

Let $W$ be the Weyl group of $\mathfrak{g}$. It is generated by simple reflections $\left\{s_{i} \mid i \in I\right\}$ obeying the relations: $s_{i}^{2}=1,\left(s_{i} s_{j}\right)^{m_{i j}}=1(i \neq j)$ where $m_{i j}=2,3,4,6$ for $\left\langle h_{i}, \alpha_{j}\right\rangle\left\langle h_{j}, \alpha_{i}\right\rangle=0,1,2,3$, respectively. Let $w_{0}$ be the longest element of $W$ and fix a reduced expression $w_{0}=s_{i_{1}} s_{i_{2}} \cdots s_{i_{l}}$. 
Then every positive root occurs exactly once in

$$
\beta_{1}=\alpha_{i_{1}}, \quad \beta_{2}=s_{i_{1}}\left(\alpha_{i_{2}}\right), \quad \ldots, \quad \beta_{l}=s_{i_{1}} s_{i_{2}} \cdots s_{i_{l-1}}\left(\alpha_{i_{l}}\right) .
$$

Correspondingly, define elements $e_{\beta_{r}} \in U_{q}(\mathfrak{g})(r=1, \ldots, l)$ by

$$
e_{\beta_{r}}=T_{i_{1}} T_{i_{2}} \cdots T_{i_{r-1}}\left(e_{i_{r}}\right) .
$$

Here $T_{i}$ is the action of the braid group on $U_{q}(\mathfrak{g})$ introduced by Lusztig [15]. It is an algebra automorphism and is given on the generators $\left\{e_{j}\right\}$ by

$$
T_{i}\left(e_{i}\right)=-k_{i} f_{i}, \quad T_{i}\left(e_{j}\right)=\sum_{r=0}^{-a_{i j}}(-1)^{r} q_{i}^{r} e_{i}^{(r)} e_{j} e_{i}^{\left(-a_{i j}-r\right)}, \quad i \neq j .
$$

$U_{q}(\mathfrak{g})$ has a subalgebra generated by $\left\{e_{i} \mid i \in I\right\}$, denoted by $U_{q}^{+}(\mathfrak{g})$. It is known that $e_{\beta_{r}} \in U_{q}^{+}(\mathfrak{g})$ holds for any $r . U_{q}^{+}(\mathfrak{g})$ has the so-called Poincaré-Birkhoff-Witt (PBW) basis. It depends on the reduced expression $s_{i_{1}} s_{i_{2}} \cdots s_{i_{l}}$ of $w_{0}$. Set $\mathbf{i}=\left(i_{1}, i_{2}, \ldots, i_{l}\right)$ and define for $A=\left(a_{1}, a_{2}, \ldots, a_{l}\right) \in\left(\mathbb{Z}_{\geq 0}\right)^{l}$

$$
E_{\mathbf{i}}^{A}=e_{\beta_{1}}^{\left(a_{1}\right)} e_{\beta_{2}}^{\left(a_{2}\right)} \cdots e_{\beta_{l}}^{\left(a_{l}\right)} .
$$

Then $\left\{E_{\mathbf{i}}^{A} \mid A \in\left(\mathbb{Z}_{\geq 0}\right)^{l}\right\}$ forms a basis of $U_{q}^{+}(\mathfrak{g})$. We hope that the notations $e_{i_{r}}$ with $i_{r} \in I$ and $e_{\beta_{r}}$ with a positive root $\beta_{r}$ can be distinguished properly from the context. In particular $e_{\beta_{r}}^{\left(a_{r}\right)}=\left(e_{\beta_{r}}\right)^{a_{r}} / \prod_{m=1}^{a_{r}} \frac{p_{r}^{m}-p_{r}^{-m}}{p_{r}-p_{r}^{-1}}$ with $p_{r}=q^{\left(\beta_{r}, \beta_{r}\right) / 2}$.

\section{Quantized algebra of functions $A_{q}(\mathfrak{g})$}

\subsection{Definition}

Following [11] we give the definition of the quantized algebra of functions $A_{q}(\mathfrak{g})$. It is valid for any symmetrizable Kac-Moody algebra $\mathfrak{g}$. Let $O_{\text {int }}(\mathfrak{g})$ be the category of integrable left $U_{q}(\mathfrak{g})$-modules $M$ such that, for any $u \in M$, there exists $l \geq 0$ satisfying $e_{i_{1}} \cdots e_{i_{l}} u=0$ for any $i_{1}, \ldots, i_{l} \in I$. Then $O_{\text {int }}(\mathfrak{g})$ is semisimple and any simple object is isomorphic to the irreducible module $V(\lambda)$ with dominant integral highest weight $\lambda$. Similarly, we can consider the category $O_{\text {int }}\left(\mathfrak{g}^{\text {opp }}\right)$ of integrable right $U_{q}(\mathfrak{g})$-modules $M^{r}$ such that, for any $v \in M^{r}$, there exists $l \geq 0$ satisfying $v f_{i_{1}} \cdots f_{i_{l}}=0$ for any $i_{1}, \ldots, i_{l} \in I$. $O_{\text {int }}\left(\mathfrak{g}^{\text {opp }}\right)$ is also semisimple and any simple object is isomorphic to the irreducible module $V^{r}(\lambda)$ with dominant integral highest weight $\lambda$. Let $u_{\lambda}$ (resp. $v_{\lambda}$ ) be a highest-weight vector of $V(\lambda)\left(\right.$ resp. $\left.V^{r}(\lambda)\right)$. Then there exists a unique bilinear form $($,

$$
V^{r}(\lambda) \otimes V(\lambda) \rightarrow \mathbb{Q}(q)
$$

satisfying

$$
\left(v_{\lambda}, u_{\lambda}\right)=1 \quad \text { and } \quad(v P, u)=(v, P u) \quad \text { for } \quad v \in V^{r}(\lambda), \quad u \in V(\lambda), \quad P \in U_{q}(\mathfrak{g}) .
$$

Let $U_{q}(\mathfrak{g})^{*}$ be $\operatorname{Hom}_{\mathbb{Q}(q)}\left(U_{q}(\mathfrak{g}), \mathbb{Q}(q)\right)$ and $\langle$,$\rangle be the canonical pairing between U_{q}(\mathfrak{g})^{*}$ and $U_{q}(\mathfrak{g})$. The comultiplication $\Delta$ of $U_{q}(\mathfrak{g})$ induces a multiplication of $U_{q}(\mathfrak{g})^{*}$ by

$$
\left\langle\varphi \varphi^{\prime}, P\right\rangle=\left\langle\varphi \otimes \varphi^{\prime}, \Delta(P)\right\rangle \quad \text { for } P \in U_{q}(\mathfrak{g}),
$$


thereby giving $U_{q}(\mathfrak{g})^{*}$ the structure of $\mathbb{Q}(q)$-algebra. It also has a $U_{q}(\mathfrak{g})$-bimodule structure by

$$
\langle x \varphi y, P\rangle=\langle\varphi, y P x\rangle \quad \text { for } x, y, P \in U_{q}(\mathfrak{g}) .
$$

We define the subalgebra $A_{q}(\mathfrak{g})$ of $U_{q}(\mathfrak{g})^{*}$ by

$$
A_{q}(\mathfrak{g})=\left\{\varphi \in U_{q}(\mathfrak{g})^{*} ; U_{q}(\mathfrak{g}) \varphi \text { belongs to } O_{\text {int }}(\mathfrak{g}) \text { and } \varphi U_{q}(\mathfrak{g}) \text { belongs to } O_{\text {int }}\left(\mathfrak{g}^{\text {opp }}\right)\right\},
$$

and call it the quantized algebra of functions.

The following theorem is the $q$-analogue of the Peter-Weyl theorem. See e.g. [11] for a proof.

Theorem 1. As a $U_{q}(\mathfrak{g})$-bimodule $A_{q}(\mathfrak{g})$ is isomorphic to $\bigoplus_{\lambda} V^{r}(\lambda) \otimes V(\lambda)$, where $\lambda$ runs over all dominant integral weights, by the homomorphisms

$$
\Psi_{\lambda}: V^{r}(\lambda) \otimes V(\lambda) \rightarrow A_{q}(\mathfrak{g})
$$

given by

$$
\left\langle\Psi_{\lambda}(v \otimes u), P\right\rangle=(v, P u)
$$

for $v \in V^{r}(\lambda), u \in V(\lambda)$, and $P \in U_{q}(\mathfrak{g})$.

Let us now assume that $\mathfrak{g}$ is a finite-dimensional simple Lie algebra. Then $A_{q}(\mathfrak{g})$ turns out a Hopf algebra. See e.g. [9, Chapter 9]. Its comultiplication is also denoted by $\Delta$.

Let $\mathcal{R}$ be the universal $R$ matrix for $U_{q}(\mathfrak{g})$. For its explicit formula see e.g. [4, p. 273]. For our purpose it is enough to know that

$$
\mathcal{R} \in q^{(\mathrm{wt} \cdot, \mathrm{wt} \cdot)} \bigoplus_{\beta \in Q^{+}}\left(U_{q}^{+}\right)_{\beta} \otimes\left(U_{q}^{-}\right)_{-\beta},
$$

where $q^{(\mathrm{wt} \cdot \mathrm{wt} \cdot)}$ is an operator acting on the tenor product $u_{\lambda} \otimes u_{\mu}$ of weight vectors $u_{\lambda}, u_{\mu}$ of weight $\lambda, \mu$ by $q^{(\mathrm{wt} \cdot \text {, wt } \cdot)}\left(u_{\lambda} \otimes u_{\mu}\right)=q^{(\lambda, \mu)} u_{\lambda} \otimes u_{\mu}, Q_{+}=\bigoplus_{i} \mathbb{Z}_{\geq 0} \alpha_{i}$, and $\left(U_{q}^{ \pm}\right)_{ \pm \beta}$ is the subspace of $U_{q}^{ \pm}(\mathfrak{g})$ spanned by root vectors corresponding to $\pm \beta$.

Fix $\lambda$, let $\left\{u_{j}^{\lambda}\right\}$ and $\left\{v_{i}^{\lambda}\right\}$ be bases of $V(\lambda)$ and $V^{r}(\lambda)$ such that $\left(v_{i}^{\lambda}, u_{j}^{\lambda}\right)=\delta_{i j}$, and $\varphi_{i j}^{\lambda}=$ $\Psi_{\lambda}\left(v_{i}^{\lambda} \otimes u_{j}^{\lambda}\right)$. Let $R$ be the so-called constant $R$ matrix for $V(\lambda) \otimes V(\mu)$. Denoting the homomorphism $U_{q}(\mathfrak{g}) \rightarrow \operatorname{End}(V(\lambda))$ by $\pi_{\lambda}$, it is given as

$$
R \propto\left(\pi_{\lambda} \otimes \pi_{\mu}\right)(\sigma \mathcal{R})
$$

where $\sigma$ stands for the exchange of the first and second components. The scalar multiple is determined appropriately depending on $\mathfrak{g}$. The reason we apply $\sigma$ is that it agrees to the convention of [18]. $R$ satisfies

$$
R \Delta(x)=\Delta^{\prime}(x) R \quad \text { for any } x \in U_{q}(\mathfrak{g}),
$$

where $\Delta^{\prime}=\sigma \circ \Delta$. Define matrix elements $R_{i j, k l}$ by $R\left(u_{k}^{\lambda} \otimes u_{l}^{\mu}\right)=\sum_{i, j} R_{i j, k l} u_{i}^{\lambda} \otimes u_{j}^{\mu}$. Define the right action of $R$ on $V^{r}(\lambda) \otimes V^{r}(\mu)$ in such a way that $\left(\left(v_{i}^{\lambda} \otimes v_{j}^{\mu}\right) R, u_{k}^{\lambda} \otimes u_{l}^{\mu}\right)=\left(v_{i}^{\lambda} \otimes v_{j}^{\mu}, R\left(u_{k}^{\lambda} \otimes u_{l}^{\mu}\right)\right)$ holds. Then we have $\left(v_{i}^{\lambda} \otimes v_{j}^{\mu}\right) R=\sum_{k, l} R_{i j, k l} v_{k}^{\lambda} \otimes v_{l}^{\mu}$. From

$$
\begin{aligned}
\sum_{m, p} R_{i j, m p}\left\langle\varphi_{m k}^{\lambda} \varphi_{p l}^{\mu}, x\right\rangle & =\sum_{m, p} R_{i j, m p}\left\langle\varphi_{m k}^{\lambda} \otimes \varphi_{p l}^{\mu}, \Delta(x)\right\rangle=\sum_{m, p} R_{i j, m p}\left(v_{m}^{\lambda} \otimes v_{p}^{\mu}, \Delta(x)\left(u_{k}^{\lambda} \otimes u_{l}^{\mu}\right)\right) \\
& =\left(\left(v_{i}^{\lambda} \otimes v_{j}^{\mu}\right) R, \Delta(x)\left(u_{k}^{\lambda} \otimes u_{l}^{\mu}\right)\right)=\left(v_{i}^{\lambda} \otimes v_{j}^{\mu}, R \Delta(x)\left(u_{k}^{\lambda} \otimes u_{l}^{\mu}\right)\right) \\
& =\sum_{m, p}\left(v_{i}^{\lambda} \otimes v_{j}^{\mu}, \Delta^{\prime}(x)\left(u_{m}^{\lambda} \otimes u_{p}^{\mu}\right)\right) R_{m p, k l}
\end{aligned}
$$




$$
\begin{aligned}
& =\sum_{m, p}\left(v_{j}^{\mu} \otimes v_{i}^{\lambda}, \Delta(x)\left(u_{p}^{\mu} \otimes u_{m}^{\lambda}\right)\right) R_{m p, k l} \\
& =\sum_{m, p}\left\langle\varphi_{j p}^{\mu} \otimes \varphi_{i m}^{\lambda}, \Delta(x)\right\rangle R_{m p, k l}=\sum_{m, p}\left\langle\varphi_{j p}^{\mu} \varphi_{i m}^{\lambda}, x\right\rangle R_{m p, k l}
\end{aligned}
$$

for any $x \in U_{q}(\mathfrak{g})$, we have

$$
\sum_{m, p} R_{i j, m p} \varphi_{m k}^{\lambda} \varphi_{p l}^{\mu}=\sum_{m, p} \varphi_{j p}^{\mu} \varphi_{i m}^{\lambda} R_{m p, k l} .
$$

We call such a relation $R T T$ relation.

\section{$3.2 \quad$ Right quotient ring $A_{q}(\mathfrak{g})_{\mathcal{S}}$}

For later use we require a certain right quotient ring of $A_{q}(\mathfrak{g})$ by a suitable multiplicatively closed subset $\mathcal{S}$. We first review the general construction from [16, Chapter 2].

Let $R$ be a noncommutative ring with 1 and $\mathcal{S}$ a multiplicatively closed subset of $R$. The following condition is called the right Ore condition:

(Ore) For any $r \in R, s \in \mathcal{S}, r \mathcal{S} \cap s R \neq \varnothing$.

Set

$$
\text { ass } \mathcal{S}=\{r \in R \mid r s=0 \text { for some } s \in \mathcal{S}\}
$$

Then under the right Ore condition ass $\mathcal{S}$ turns out a two-sided ideal. Let ${ }^{-}: R \rightarrow R /$ ass $\mathcal{S}$ denote the canonical projection. Suppose

(reg) $\overline{\mathcal{S}}$ consists of regular elements, namely, elements $x$ such that both $x r=0$ and $r x=0$ imply $r=0$.

Then a theorem in [16, Chapter 2] states

Theorem 2 (Theorem 2.1 .12 of [16]). The right quotient ring $R_{\mathcal{S}}$ exists, if and only if (Ore) and (reg) are satisfied.

By passing to the images by ${ }^{-}$, it suffices to consider the case when ass $\mathcal{S}=0$, and then elements of $R_{\mathcal{S}}$ are of the form $r / s$. For $r_{i} / s_{i} \in R / \mathcal{S}(i=1,2)$ the addition and multiplication formulae are given by

$$
r_{1} / s_{1}+r_{2} / s_{2}=\left(r_{1} u+r_{2} u^{\prime}\right) /\left(s_{1} u\right), \quad\left(r_{1} / s_{1}\right)\left(r_{2} / s_{2}\right)=\left(r_{1} v^{\prime}\right) /\left(s_{2} v\right),
$$

where $u, u^{\prime}, v, v^{\prime}$ are so chosen that $s_{1} u=s_{2} u^{\prime}\left(u \in \mathcal{S}, u^{\prime} \in R\right), r_{2} v=s_{1} v^{\prime}\left(v \in \mathcal{S}, v^{\prime} \in R\right)$.

Let us return to our case where $R=A_{q}(\mathfrak{g})$.

Definition 1. For any $i \in I$, let $u_{w_{0} \varpi_{i}}$ (resp. $v_{\varpi_{i}}$ ) be a lowest (resp. highest) weight vector of $V\left(\varpi_{i}\right)\left(\operatorname{resp} . V^{r}\left(\varpi_{i}\right)\right)$. Set

$$
\sigma_{i}=\Psi_{\varpi_{i}}\left(v_{\varpi_{i}} \otimes u_{w_{0} \varpi_{i}}\right) .
$$

The following proposition is proven in [9]. However, we dare to prove again, since conventions might be different.

Proposition 1 (Corollary 9.1.4 of [9]). Let $\varphi_{\lambda \mu}$ be an element of $A_{q}(\mathfrak{g})$ such that $k_{i} \varphi_{\lambda \mu}=$ $q_{i}^{\left\langle h_{i}, \mu\right\rangle} \varphi_{\lambda \mu}, \varphi_{\lambda \mu} k_{i}=q_{i}^{\left\langle h_{i}, \lambda\right\rangle} \varphi_{\lambda \mu}$ for any $i \in I$. Then the following commutation relation holds:

$$
q^{\left(\varpi_{i}, \lambda\right)} \sigma_{i} \varphi_{\lambda \mu}=q^{\left(w_{0} \varpi_{i}, \mu\right)} \varphi_{\lambda \mu} \sigma_{i}
$$

In particular, $\sigma_{i} \sigma_{j}=\sigma_{j} \sigma_{i}$ for any $i, j$. 
Proof. Without loss of generality one can assume $\varphi_{\lambda \mu}=\Psi_{\nu}\left(v_{\lambda} \otimes u_{\mu}\right)$ for some $\nu, v_{\lambda} \in V^{r}(\nu)$, $u_{\mu} \in V(\nu)$ such that $k_{i} u_{\mu}=q_{i}^{\left\langle h_{i}, \mu\right\rangle} u_{\mu}, v_{\lambda} k_{i}=q_{i}^{\left\langle h_{i}, \lambda\right\rangle} v_{\lambda}$. In view of (9), (10) we have

$$
R\left(u_{w_{0} \varpi_{i}} \otimes u_{\mu}\right)=q^{\left(w_{0} \varpi_{i}, \mu\right)} u_{w_{0} \varpi_{i}} \otimes u_{\mu}, \quad\left(v_{\varpi_{i}} \otimes v_{\lambda}\right) R=q^{\left(\varpi_{i}, \lambda\right)} v_{\varpi_{i}} \otimes v_{\lambda} .
$$

Then (11) implies the commutation relation. The second relation follows from the first one, since $\left(\varpi_{i}, \varpi_{i}\right)=\left(w_{0} \varpi_{i}, w_{0} \varpi_{i}\right)$.

Let $n$ be the rank of $\mathfrak{g}$ and define

$$
\mathcal{S}=\left\{\sigma_{1}^{m_{1}} \cdots \sigma_{n}^{m_{n}} \mid m_{1}, \ldots, m_{n} \in \mathbb{Z}_{\geq 0}\right\},
$$

which is obviously multiplicatively closed subset of $A_{q}(\mathfrak{g})$.

Lemma 1. Let $s$ be a nonzero element in $\operatorname{Im} \Psi_{\lambda}$ satisfying $f_{i} s=s f_{i}=0$ for any $i \in I$. Then $s \in \mathbb{Q}(q)^{\times} \sigma_{1}^{\lambda_{1}} \cdots \sigma_{n}^{\lambda_{n}}$ where $\mathbb{Q}(q)^{\times}=\mathbb{Q}(q) \backslash\{0\}$ and $\lambda_{i}=\left\langle h_{i}, \lambda\right\rangle$.

Proof. By (7), (8) $f_{i} \sigma_{1}^{\lambda_{1}} \cdots \sigma_{n}^{\lambda_{n}}=\sigma_{1}^{\lambda_{1}} \cdots \sigma_{n}^{\lambda_{n}} f_{i}=0$ for any $i$ and $\sigma_{1}^{\lambda_{1}} \cdots \sigma_{n}^{\lambda_{n}}$ belongs to $\operatorname{Im} \Psi_{\lambda}$. By Theorem 1 such an element is unique up to an element of $\mathbb{Q}(q)^{\times}$.

In particular $\sigma_{i}$ is characterized as the unique element (up to an overall constant) in $\operatorname{Im} \Psi_{\varpi_{i}}$ such that $f_{j} \sigma_{i}=\sigma_{i} f_{j}=0$ for all $j \in I$. We remark that Theorem 1 implies that if a nonzero element $\varphi_{\lambda, \mu} \in A_{q}(\mathfrak{g})$ satisfies the assumption of Proposition 1 and $f_{j} \varphi_{\lambda, \mu}=\varphi_{\lambda, \mu} f_{j}=0$ for all $j$, then $\lambda=w_{0} \mu$ must hold.

In [9] it is shown that $A_{q}(\mathfrak{g})$ is an integral domain (Lemma 9.1.9), hence (reg) is satisfied, and that (Ore) is also satisfied (Lemma 9.1.10). Therefore we have the following theorem. (A proof is attached for self-containedness.)

Theorem 3. The right quotient ring $A_{q}(\mathfrak{g})_{\mathcal{S}}$ exists.

Proof. In view of Theorem 2 it is enough to show that

(1) if $\varphi \neq 0$, then $\varphi s \neq 0$ for any $s \in \mathcal{S}$,

$\left(1^{\prime}\right)$ if $\varphi \neq 0$, then $s \varphi \neq 0$ for any $s \in \mathcal{S}$, and

(2) the right Ore condition is satisfied,

since (1) implies ass $\mathcal{S}=0$, then (1) and $\left(1^{\prime}\right)$ imply $\overline{\mathcal{S}}=\mathcal{S}$ consists of regular elements.

Let us prove (1). Let $\varphi=\sum_{j} \varphi_{j}$ be the two-sided weight decomposition. If $\varphi_{j} s \neq 0$ for some $j, \varphi s \neq 0$ since the weights of $\varphi_{j} s$ are distinct. Hence we can reduce the claim when $\varphi$ is a weight vector. Suppose $\varphi=\sum_{\mu} \varphi_{\mu}, \varphi_{\mu} \in \operatorname{Im} \Psi_{\mu}$ and let $\lambda$ be a maximal weight, with respect to the standard ordering on weights, such that $\varphi_{\lambda} \neq 0$. Choose sequences $i_{1}, \ldots, i_{k}$ and $j_{1}, \ldots, j_{l}$ such that $f_{i_{k}} \cdots f_{i_{1}} \varphi_{\lambda} f_{j_{1}} \cdots f_{j_{l}}$ turns out a left-lowest and right-highest weight vector. Then by Lemma 1 it coincides with $c s^{\prime}$ with some $c \in \mathbb{Q}(q)^{\times}, s^{\prime} \in \mathcal{S}$. Then

$$
f_{i_{k}} \cdots f_{i_{1}}(\varphi s) f_{j_{1}} \cdots f_{j_{l}}=c^{\prime} s^{\prime} s+\cdots
$$

with another $c^{\prime} \in \mathbb{Q}(q)^{\times}$. By the maximality of $\lambda$ the remaining part $+\cdots$ in the right-hand side does not contain the terms with the same two-sided weight. Hence $\cdots=0$. Therefore, the left-hand side is not 0 and we conclude $\varphi s \neq 0$.

$\left(1^{\prime}\right)$ is similar. For $(2)$ we can reduce the claim when $\varphi$ is a weight vector, and in this case the claim is clear from Proposition 1. 


\subsection{Realization by generators and relations}

We consider the fundamental representation $V\left(\varpi_{1}\right)$ of $U_{q}(\mathfrak{g})$ for $\mathfrak{g}=A_{n-1}, C_{n}, G_{2}$. Set $N=$ $\operatorname{dim} V\left(\varpi_{1}\right)$. It is known [5,18] that $A_{q}(\mathfrak{g})$ for $\mathfrak{g}=A_{n-1}, C_{n}, G_{2}$ is realized as an associative algebra with appropriate generators $\left(t_{i j}\right)_{1 \leq i, j \leq N}$ corresponding to $V^{r}\left(\varpi_{1}\right) \otimes V\left(\varpi_{1}\right)$ satisfying $R T T$ relations

$$
\sum_{m, p} R_{i j, m p} t_{m k} t_{p l}=\sum_{m, p} t_{j p} t_{i m} R_{m p, k l},
$$

and additional ones depending on $\mathfrak{g}$. See below for each $\mathfrak{g}$ under consideration. In all cases, there exists a comultiplication $\Delta: A_{q} \rightarrow A_{q} \otimes A_{q}$ given by

$$
\Delta\left(t_{i j}\right)=\sum_{k} t_{i k} \otimes t_{k j}
$$

\subsection{1 $\quad A_{n-1}$ case}

We present formulae for $A_{q}\left(A_{n-1}\right)$. In this case $N=n$. Let $u_{1}$ and $v_{1}$ be the highest-weight vectors of $V\left(\varpi_{1}\right)$ and $V^{r}\left(\varpi_{1}\right)$ such that $\left(v_{1}, u_{1}\right)=1$ and set $u_{j}=f_{j-1} f_{j-2} \cdots f_{1} u_{1}, v_{j}=$ $v_{1} e_{1} e_{2} \cdots e_{j-1}$ for $2 \leq j \leq n$. Then the constant $R$ matrix is given by

$$
\sum_{i, j, k, l} R_{i j, k l} E_{i k} \otimes E_{j l}=q \sum_{i} E_{i i} \otimes E_{i i}+\sum_{i \neq j} E_{i i} \otimes E_{j j}+\left(q-q^{-1}\right) \sum_{i>j} E_{i j} \otimes E_{j i},
$$

where $E_{i j}$ is the matrix unit. Define $t_{i j}=\Psi_{\varpi_{1}}\left(v_{i} \otimes u_{j}\right)$. Then the RTT relations among $\left(t_{i j}\right)_{1 \leq i, j \leq N}$ read explicitly as follows

$$
\begin{aligned}
& {\left[t_{i k}, t_{j l}\right]= \begin{cases}0, & i<j, k>l, \\
\left(q-q^{-1}\right) t_{j k} t_{i l}, & i<j, k<l,\end{cases} } \\
& t_{i k} t_{j k}=q t_{j k} t_{i k}, \quad i<j, \quad t_{k i} t_{k j}=q t_{k j} t_{k i}, \quad i<j .
\end{aligned}
$$

In $A_{n-1}$ case we need another condition that the quantum determinant is 1, i.e.,

$$
\sum_{\sigma \in \mathfrak{S}_{n}}(-q)^{\ell(\sigma)} t_{1 \sigma_{1}} \cdots t_{n \sigma_{n}}=1
$$

where $\mathfrak{S}_{n}=W\left(A_{n-1}\right)$ is the symmetric group of degree $n$ and $\ell(\sigma)$ is the length of $\sigma$.

According to Definition 1 , we have $\sigma_{1}=t_{13}$ and $\sigma_{2}=t_{12} t_{23}-q t_{22} t_{13}$. As an exposition, we note that $\sigma_{i} e_{i}$ in (39) is derived from

$$
\begin{aligned}
\left\langle\sigma_{1} e_{1}, P\right\rangle & =\left\langle t_{13} e_{1}, P\right\rangle=\left(v_{1} e_{1}, P u_{3}\right)=\left(v_{2}, P u_{3}\right)=\left\langle t_{23}, P\right\rangle, \\
\left\langle\sigma_{2} e_{2}, P\right\rangle & =\left\langle\left(t_{12} \otimes t_{23}-q t_{22} \otimes t_{13}\right) \Delta\left(e_{2}\right), \Delta(P)\right\rangle=\left\langle t_{12} k_{2} \otimes t_{23} e_{2}-q t_{22} e_{2} \otimes t_{13}, \Delta(P)\right\rangle \\
& =\left\langle t_{12} \otimes t_{33}-q t_{32} \otimes t_{13}, \Delta(P)\right\rangle=\left\langle t_{12} t_{33}-q t_{32} t_{13}, P\right\rangle
\end{aligned}
$$

for any $P \in U_{q}\left(A_{2}\right)$. See e.g. [17] for an extensive treatment.

\subsection{2 $C_{n}$ case}

We present formulae for $A_{q}\left(C_{n}\right)$. In this case $N=2 n$. Let $u_{1}$ be the highest-weight vector of $V\left(\varpi_{1}\right)$ and define $u_{j}$ for $2 \leq j \leq 2 n$ recursively by $u_{j+1}=f_{j} u_{j}(j \leq n),-f_{2 n-j} u_{j}(j>n)$. Let $\left\{v_{i}\right\}$ be the dual basis to $\left\{u_{i}\right\}$ in $V^{r}\left(\varpi_{1}\right)$, namely, $\left\{v_{i}\right\}$ are determined by $\left(v_{i}, u_{j}\right)=\delta_{i j}$. Then the constant $R$ matrix is given by

$$
\sum_{i, j, k, l} R_{i j, k l} E_{i k} \otimes E_{j l}=q \sum_{i} E_{i i} \otimes E_{i i}+\sum_{i \neq j, j^{\prime}} E_{i i} \otimes E_{j j}+q^{-1} \sum_{i} E_{i i} \otimes E_{i^{\prime} i^{\prime}}
$$




$$
\begin{aligned}
& +\left(q-q^{-1}\right) \sum_{i>j} E_{i j} \otimes E_{j i}-\left(q-q^{-1}\right) \sum_{i>j} \epsilon_{i} \epsilon_{j} q^{\varrho_{i}-\varrho_{j}} E_{i j} \otimes E_{i^{\prime} j^{\prime}}, \\
& i^{\prime}=2 n+1-i, \quad \epsilon_{i}=1, \quad 1 \leq i \leq n, \quad \epsilon_{i}=-1, \quad n<i \leq 2 n, \\
& \left(\varrho_{1}, \ldots, \varrho_{2 n}\right)=(n-1, n-2, \ldots, 1,0,0,-1, \ldots,-n+1) .
\end{aligned}
$$

Define $t_{i j}=\Psi_{\varpi_{1}}\left(v_{i} \otimes u_{j}\right)$. The $R T T$ relations are given by (13) with the above $R_{i j, k l}$. Additional relations are given by

$$
\sum_{j, k, l} C_{j k} C_{l m} t_{i j} t_{l k}=\sum_{j, k, l} C_{i j} C_{k l} t_{k j} t_{l m}=-\delta_{i m}, \quad C_{i j}=\delta_{i, j^{\prime}} \epsilon_{i} q^{\varrho_{j}} .
$$

\subsection{3 $\quad G_{2}$ case}

We have $N=7$ in this case. We adopt the basis $\left\{u_{i}\right\}$ of $V\left(\varpi_{1}\right)$ that has the representation matrices given as in [19, equation (29)], and let $\left\{v_{i}\right\}$ the dual basis in $V^{r}\left(\varpi_{1}\right)$. Define $t_{i j}=$ $\Psi_{\varpi_{1}}\left(v_{i} \otimes u_{j}\right)$. Then $A_{q}\left(G_{2}\right)$ is generated by $\left(t_{i j}\right)_{1 \leq i, j \leq 7}$ satisfying (i) and (ii) given below.

(i) RTT relations (13) with the structure constants specified by $R_{i j, k l}=R_{k l}^{i j}$ in [19, equation (33)].

(ii) Additional relations

$$
g^{i j}=\sum_{k, l} t_{j l} t_{i k} g^{k l}, \quad \sum_{k} f_{k}^{i j} t_{k m}=\sum_{k, l} t_{j l} t_{i k} f_{m}^{k l},
$$

where $g^{i j}$ and $f_{k}^{i j}$ are given by [19, equations (30), (31)].

The relations [19, equations (20), (22)] are equivalent to (15) if the $R T T$ relations are imposed. See the explanation after [19, Definition 7]. Note also that we use the opposite indices of the Dynkin diagram to [19].

\section{Representations of $A_{q}(\mathfrak{g})$}

\subsection{General remarks}

Let us recall the results in $[22,23]$ on the representations of $A_{q}(\mathfrak{g})$ necessary in this paper. Consider the simplest example $A_{q}\left(A_{1}\right)$ generated by $t_{11}, t_{12}, t_{21}, t_{22}$ with the relations

$$
\begin{aligned}
& t_{11} t_{21}=q t_{21} t_{11}, \quad t_{12} t_{22}=q t_{22} t_{12}, \quad t_{11} t_{12}=q t_{12} t_{11}, \quad t_{21} t_{22}=q t_{22} t_{21}, \\
& {\left[t_{12}, t_{21}\right]=0, \quad\left[t_{11}, t_{22}\right]=\left(q-q^{-1}\right) t_{21} t_{12}, \quad t_{11} t_{22}-q t_{12} t_{21}=1 .}
\end{aligned}
$$

Let $\mathrm{Osc}_{q}=\left\langle\mathbf{a}^{+}, \mathbf{a}^{-}, \mathbf{k}\right\rangle$ be the $q$-oscillator algebra, i.e., an associative algebra with the relations

$$
\mathbf{k a}^{+}=q \mathbf{a}^{+} \mathbf{k}, \quad \mathbf{k} \mathbf{a}^{-}=q^{-1} \mathbf{a}^{-} \mathbf{k}, \quad \mathbf{a}^{-} \mathbf{a}^{+}=\mathbf{1}-q^{2} \mathbf{k}^{2}, \quad \mathbf{a}^{+} \mathbf{a}^{-}=\mathbf{1}-\mathbf{k}^{2} .
$$

It has a representation on the Fock space $\mathcal{F}_{q}=\bigoplus_{m \geq 0} \mathbb{C}(q)|m\rangle$ :

$$
\mathbf{k}|m\rangle=q^{m}|m\rangle, \quad \mathbf{a}^{+}|m\rangle=|m+1\rangle, \quad \mathbf{a}^{-}|m\rangle=\left(1-q^{2 m}\right)|m-1\rangle .
$$

In what follows, the symbols $\mathbf{k}, \mathbf{a}^{+}, \mathbf{a}^{-}$shall also be regarded as the elements from $\operatorname{End}\left(\mathcal{F}_{q}\right)$. It is easy to check that the following map $\pi$ defines an irreducible representation of $A_{q}\left(A_{1}\right)$ on $\mathcal{F}_{q}$ :

$$
\pi:\left(\begin{array}{cc}
t_{11} & t_{12} \\
t_{21} & t_{22}
\end{array}\right) \mapsto\left(\begin{array}{cc}
\mu \mathbf{a}^{-} & \alpha \mathbf{k} \\
-q \alpha^{-1} \mathbf{k} & \mu^{-1} \mathbf{a}^{+}
\end{array}\right),
$$

where $\alpha, \mu$ are nonzero parameters. 
Theorem 4 ([22, 23]).

(1) For each vertex $i$ of the Dynkin diagram of $\mathfrak{g}, A_{q}(\mathfrak{g})$ has an irreducible representation $\pi_{i}$ factoring through $(18)$ via $A_{q}(\mathfrak{g}) \rightarrow A_{q_{i}}\left(s l_{2, i}\right)$. (sl $l_{2, i}$ denotes the sl $l_{2}$-subalgebra of $\mathfrak{g}$ associated to $i$.)

(2) Irreducible representations of $A_{q}(\mathfrak{g})$ are in one to one correspondence with the elements of the Weyl group $W$ of $\mathfrak{g}$.

(3) Let $w=s_{i_{1}} \cdots s_{i_{l}} \in W$ be an reduced expression in terms of the simple reflections. Then the irreducible representation corresponding to $w$ is isomorphic to $\pi_{i_{1}} \otimes \cdots \otimes \pi_{i_{l}}$.

Actually the assertions (2) and (3) hold up to the degrees of freedom of the parameters $\alpha, \mu$ in (18). See [22] for the detail. We call $\pi_{i}(i=1, \ldots$, rank $\mathfrak{g})$ the fundamental representations. For simplicity we denote $\pi_{i_{1}} \otimes \cdots \otimes \pi_{i_{l}}$ by $\pi_{i_{1}, \ldots, i_{l}}$.

A crucial corollary of Theorem 4 is the following:

$$
\text { If } s_{i_{1}} \cdots s_{i_{l}}=s_{j_{1}} \cdots s_{j_{l}} \in W \text { are reduced expressions, then } \pi_{i_{1}, \ldots, i_{l}} \simeq \pi_{j_{1}, \ldots, j_{l}} \text {. }
$$

In particular, there exists the isomorphism $\Phi: \mathcal{F}_{q_{i_{1}}} \otimes \cdots \otimes \mathcal{F}_{q_{i_{l}}} \rightarrow \mathcal{F}_{q_{j_{1}}} \otimes \cdots \otimes \mathcal{F}_{q_{j_{l}}}$ characterized (up to an overall constant) by

$$
\pi_{j_{1}, \ldots, j_{l}}(g) \circ \Phi=\Phi \circ \pi_{i_{1}, \ldots, i_{l}}(g) \quad \forall g \in A_{q}(\mathfrak{g}) .
$$

Here $\pi_{i_{1}, \ldots, i_{l}}\left(g=t_{i j}\right)$ for example means the tensor product representation $\sum_{r_{1}, \ldots, r_{l-1}} \pi_{i_{1}}\left(t_{i r_{1}}\right) \otimes$ $\cdots \otimes \pi_{i_{l}}\left(t_{r_{l-1}, j}\right)$ obtained by the $(l-1)$-fold application of the coproduct $(14)$.

Elements of the Fock space $\left|m_{1}\right\rangle \otimes \cdots \otimes\left|m_{l}\right\rangle \in \mathcal{F}_{q_{j_{1}}} \otimes \cdots \otimes \mathcal{F}_{q_{j_{l}}}$ will simply be denoted by $\left|m_{1}, \ldots, m_{l}\right\rangle$. We will always normalize the intertwiner by the condition $\Phi|0,0, \ldots, 0\rangle=$ $|0,0, \ldots, 0\rangle$. The exchange of the $i$ th and the $j$ th tensor components from the left will be denoted by $P_{i j}$. In the remainder of this section we concentrate on $A_{q}(\mathfrak{g})$ of rank 2 cases $\mathfrak{g}=A_{2}, C_{2}$ and $G_{2}$, and present the concrete forms of the fundamental representations, definition of the intertwiners with a few examples of their matrix elements.

\section{$4.2 \quad A_{2}$ case}

Let $T=\left(t_{i j}\right)_{1 \leq i, j \leq 3}$ be the $3 \times 3$ matrix of the generators of $A_{q}\left(A_{2}\right)$. The fundamental representations $\pi_{i}: A_{q}\left(A_{2}\right) \rightarrow \operatorname{End}\left(\mathcal{F}_{q}\right)(i=1,2)$ are given by

$$
\pi_{1}(T)=\left(\begin{array}{ccc}
\mu_{1} \mathbf{a}^{-} & \alpha_{1} \mathbf{k} & 0 \\
-q \alpha_{1}^{-1} \mathbf{k} & \mu_{1}^{-1} \mathbf{a}^{+} & 0 \\
0 & 0 & 1
\end{array}\right), \quad \pi_{2}(T)=\left(\begin{array}{ccc}
1 & 0 & 0 \\
0 & \mu_{2} \mathbf{a}^{-} & \alpha_{2} \mathbf{k} \\
0 & -q \alpha_{2}^{-1} \mathbf{k} & \mu_{2}^{-1} \mathbf{a}^{+}
\end{array}\right)
$$

where $\alpha_{i}, \mu_{i}$ are nonzero parameters.

The Weyl group $W=\left\langle s_{1}, s_{2}\right\rangle$ is the Coxeter system with the relations

$$
s_{1}^{2}=s_{2}^{2}=1, \quad s_{1} s_{2} s_{1}=s_{2} s_{1} s_{2} .
$$

Thus we have the isomorphism $\pi_{121} \simeq \pi_{212}$. Let $\Phi$ be the corresponding intertwiner and denote by $R$ the checked intertwiner $\Phi^{\vee}$ explained after (1)

$$
\pi_{121} \Phi=\Phi \pi_{212}, \quad \pi_{121} R=R \pi_{212}^{\prime}, \quad \pi_{212}^{\prime}=P_{13} \pi_{212} P_{13}, \quad R=\Phi P_{13} \in \operatorname{End}\left(\mathcal{F}_{q}^{\otimes 3}\right) .
$$

For example $\pi_{212}^{\prime}\left(t_{i j}\right)=\sum_{k, l} \pi_{2}\left(t_{l, j}\right) \otimes \pi_{1}\left(t_{k, l}\right) \otimes \pi_{2}\left(t_{i k}\right)$. Define the matrix elements of $R$ and its parameter-free part $\mathcal{R}$ by

$$
R|i, j, k\rangle=\sum_{a, b, c} R_{i j k}^{a b c}|a, b, c\rangle, \quad R_{i j k}^{a b c}=\mu_{1}^{a-j+k} \mu_{2}^{b-a-k} \mathcal{R}_{i j k}^{a b c} .
$$


Then the following properties are valid for $\mathcal{R}=\left(\mathcal{R}_{i j k}^{a b c}\right)[13]$ :

$$
\begin{aligned}
& \mathcal{R}_{i j k}^{a b c} \in \mathbb{Z}[q], \quad \mathcal{R}_{i j k}^{a b c}=0 \quad \text { unless } \quad(a+b, b+c)=(i+j, j+k), \\
& \mathcal{R}^{-1}=\mathcal{R}, \quad \mathcal{R}_{i j k}^{a b c}=\mathcal{R}_{k j i}^{c b a}, \quad \mathcal{R}_{i j k}^{a b c}=\frac{\left(q^{2}\right)_{i}\left(q^{2}\right)_{j}\left(q^{2}\right)_{k}}{\left(q^{2}\right)_{a}\left(q^{2}\right)_{b}\left(q^{2}\right)_{c}} \mathcal{R}_{a b c}^{i j k}, \\
& \left.\mathcal{R}_{i j k}^{a b c}\right|_{q=0}=\delta_{i, b+(a-c)_{+}} \delta_{j, \min (a, c)} \delta_{k, b+(c-a)_{+}} \cdot
\end{aligned}
$$

Here $\left(q^{2}\right)_{a}=\prod_{m=1}^{a}\left(1-q^{2 m}\right)$ and $(y)_{+}=\max (0, y)$. Due to $(20), \mathcal{R}$ is the infinite direct sum of finite-dimensional matrices. An explicit formula of $\mathcal{R}_{i j k}^{a b c}$ was obtained in [10] (unfortunately with misprint) and in [1, equation (59)] (in a different context and gauge including square roots). The formula exactly matching the present convention is [13, equation (2.20)]. The $\mathcal{R}$ satisfies [10] the tetrahedron equation (2).

Example 1. The following is the list of all the nonzero $\mathcal{R}_{314}^{a b c}$ :

$$
\begin{aligned}
& \mathcal{R}_{314}^{041}=-q^{2}\left(1-q^{4}\right)\left(1-q^{6}\right)\left(1-q^{8}\right), \\
& \mathcal{R}_{314}^{132}=\left(1-q^{6}\right)\left(1-q^{8}\right)\left(1-q^{4}-q^{6}-q^{8}-q^{10}\right), \\
& \mathcal{R}_{314}^{223}=q^{2}\left(1+q^{2}\right)\left(1+q^{4}\right)\left(1-q^{6}\right)\left(1-q^{6}-q^{10}\right), \\
& \mathcal{R}_{314}^{314}=q^{6}\left(1+q^{2}+q^{4}-q^{8}-q^{10}-q^{12}-q^{14}\right), \\
& \mathcal{R}_{314}^{405}=q^{12} .
\end{aligned}
$$

Thus $\left.\mathcal{R}_{314}^{a b c}\right|_{q=0}=\delta_{a, 1} \delta_{b, 3} \delta_{c, 2}$ in agreement with (22).

\section{$4.3 \quad C_{2}$ case}

We have $\left(q_{1}, q_{2}\right)=\left(q, q^{2}\right)$. Let $T=\left(t_{i j}\right)_{1 \leq i, j \leq 4}$ be the $4 \times 4$ matrix of the generators of $A_{q}\left(C_{2}\right)$. We use $\operatorname{Osc}_{q^{2}}=\left\langle\mathbf{A}^{+}, \mathbf{A}^{-}, \mathbf{K}\right\rangle$ in addition to $\mathrm{Osc}_{q}=\left\langle\mathbf{a}^{+}, \mathbf{a}^{-}, \mathbf{k}\right\rangle$ (16). The fundamental representations $\pi_{i}: A_{q}\left(C_{2}\right) \rightarrow \operatorname{End}\left(\mathcal{F}_{q_{i}}\right)(i=1,2)$ are given by

$$
\begin{aligned}
\pi_{1}(T) & =\left(\begin{array}{cccc}
\mu_{1} \mathbf{a}^{-} & \alpha_{1} \mathbf{k} & 0 & 0 \\
-q \alpha_{1}^{-1} \mathbf{k} & \mu_{1}^{-1} \mathbf{a}^{+} & 0 & 0 \\
0 & 0 & \mu_{1} \mathbf{a}^{-} & -\alpha_{1} \mathbf{k} \\
0 & 0 & q \alpha_{1}^{-1} \mathbf{k} & \mu_{1}^{-1} \mathbf{a}^{+}
\end{array}\right) \\
\pi_{2}(T) & =\left(\begin{array}{cccc}
1 & 0 & 0 & 0 \\
0 & \mu_{2} \mathbf{A}^{-} & \alpha_{2} \mathbf{K} & 0 \\
0 & -q^{2} \alpha_{2}^{-1} \mathbf{K} & \mu_{2}^{-1} \mathbf{A}^{+} & 0 \\
0 & 0 & 0 & 1
\end{array}\right)
\end{aligned}
$$

where $\alpha_{i}, \mu_{i}$ are nonzero parameters.

The Weyl group $W=\left\langle s_{1}, s_{2}\right\rangle$ is the Coxeter system with the relations

$$
s_{1}^{2}=s_{2}^{2}=1, \quad s_{2} s_{1} s_{2} s_{1}=s_{1} s_{2} s_{1} s_{2} .
$$

Thus we have the isomorphism $\pi_{2121} \simeq \pi_{1212}$. Let $\Phi$ be the corresponding intertwiner and denote by $K$ the checked intertwiner $\Phi^{\vee}$

$$
\begin{aligned}
& \pi_{2121} \Phi=\Phi \pi_{1212}, \quad \pi_{2121} K=K \pi_{2121}^{\prime}, \quad \pi_{2121}^{\prime}=P_{14} P_{23} \pi_{1212} P_{14} P_{23} \\
& K=\Phi P_{14} P_{23} \in \operatorname{End}\left(\mathcal{F}_{q^{2}} \otimes \mathcal{F}_{q} \otimes \mathcal{F}_{q^{2}} \otimes \mathcal{F}_{q}\right) .
\end{aligned}
$$


Define the matrix elements of $K$ and its parameter-free part $\mathcal{K}$ by

$$
K|i, j, k, l\rangle=\sum_{a, b, c, d} K_{i j k l}^{a b c d}|a, b, c, d\rangle, \quad K_{i j k l}^{a b c d}=\mu_{1}^{2(c-k)} \mu_{2}^{b-j} \mathcal{K}_{i j k l}^{a b c d} .
$$

Then the following properties are valid for $\mathcal{K}=\left(\mathcal{K}_{i j k l}^{a b c d}\right)[13]$ :

$$
\begin{aligned}
& \mathcal{K}_{i j k l}^{a b c d} \in \mathbb{Z}[q], \quad \mathcal{K}_{i j k l}^{a b c d}=0 \quad \text { unless }(a+b+c, b+2 c+d)=(i+j+k, j+2 k+l), \\
& \mathcal{K}^{-1}=\mathcal{K}, \quad \mathcal{K}_{i j k l}^{a b c d}=\frac{\left(q^{4}\right)_{i}\left(q^{2}\right)_{j}\left(q^{4}\right)_{k}\left(q^{2}\right)_{l}}{\left(q^{4}\right)_{a}\left(q^{2}\right)_{b}\left(q^{4}\right)_{c}\left(q^{2}\right)_{d}} \mathcal{K}_{a b c d}^{i j k l}, \\
& \left.\mathcal{K}_{i j k l}^{a b c d}\right|_{q=0}=\delta_{i, a^{\prime}} \delta_{j, b^{\prime}} \delta_{k, c^{\prime}} \delta_{l, d^{\prime}}, \\
& a^{\prime}=x+a+b-d, \quad b^{\prime}=c+d-x-\min (a, c+x), \\
& c^{\prime}=\min (a, c+x), \quad d^{\prime}=b+(c-a+x)_{+}, \quad x=\left(c-a+(d-b)_{+}\right)_{+} .
\end{aligned}
$$

Due to $(24), \mathcal{K}$ is the infinite direct sum of finite-dimensional matrices. An explicit formula of $\mathcal{K}_{i j k l}^{a b c d}$ is available in [13, equations (3.27), (3.28)]. This $\mathcal{K}$ and $\mathcal{R}$ in Section 4.2 satisfy [13] the $3 \mathrm{D}$ reflection equation (3).

Example 2. The following is the list of all the nonzero $\mathcal{K}_{2110}^{a b c d}$ :

$$
\begin{aligned}
& \mathcal{K}_{2110}^{1300}=q^{8}\left(1-q^{8}\right), \\
& \mathcal{K}_{2110}^{2110}=-q^{4}\left(1-q^{8}+q^{14}\right), \\
& \mathcal{K}_{2110}^{2201}=-q^{6}\left(1+q^{2}\right)\left(1-q^{2}+q^{4}-q^{6}-q^{10}\right), \\
& \mathcal{K}_{2110}^{3011}=1-q^{8}+q^{14}, \\
& \mathcal{K}_{2110}^{3102}=-q^{10}\left(1-q+q^{2}\right)\left(1+q+q^{2}\right), \\
& \mathcal{K}_{2110}^{4003}=q^{4} .
\end{aligned}
$$

Thus $\left.\mathcal{K}_{2110}^{a b c d}\right|_{q=0}=\delta_{a, 3} \delta_{b, 0} \delta_{c, 1} \delta_{d, 1}$ in agreement with (26).

\section{$4.4 G_{2}$ case}

We have $\left(q_{1}, q_{2}\right)=\left(q, q^{3}\right)$. Let $T=\left(t_{i j}\right)_{1 \leq i, j \leq 7}$ be the $7 \times 7$ matrix of the generators of $A_{q}\left(G_{2}\right)$. We use $\operatorname{Osc}_{q^{3}}=\left\langle\mathbf{A}^{+}, \mathbf{A}^{-}, \mathbf{K}\right\rangle$ in addition to $\operatorname{Osc}_{q}=\left\langle\mathbf{a}^{+}, \mathbf{a}^{-}, \mathbf{k}\right\rangle$ (16). The fundamental representations $\pi_{i}: A_{q}\left(G_{2}\right) \rightarrow \operatorname{End}\left(\mathcal{F}_{q_{i}}\right)(i=1,2)$ are given by

$$
\begin{aligned}
& \pi_{1}(T)=\left(\begin{array}{ccccccc}
\mu_{1} \mathbf{a}^{-} & \alpha_{1} \mathbf{k} & 0 & 0 & 0 & 0 & 0 \\
-q \alpha_{1}^{-1} \mathbf{k} \mu_{1}^{-1} \mathbf{a}^{+} & 0 & 0 & 0 & 0 & 0 \\
0 & 0 & \left(\mu_{1} \mathbf{a}^{-}\right)^{2} & {[2]_{1} \alpha_{1} \mu_{1} \mathbf{k} \mathbf{a}^{-}} & \left(\alpha_{1} \mathbf{k}\right)^{2} & 0 & 0 \\
0 & 0 & -q \alpha_{1}^{-1} \mu_{1} \mathbf{a}^{-} \mathbf{k} & \mathbf{a}^{-} \mathbf{a}^{+}-\mathbf{k}^{2} & \alpha_{1} \mu_{1}^{-1} \mathbf{k} \mathbf{a}^{+} & 0 & 0 \\
0 & 0 & \left(q \alpha_{1}^{-1} \mathbf{k}\right)^{2} & -[2]_{1}\left(\alpha_{1} \mu_{1}\right)^{-1} \mathbf{k} \mathbf{a}^{+} & \left(\mu_{1}^{-1} \mathbf{a}^{+}\right)^{2} & 0 & 0 \\
0 & 0 & 0 & 0 & 0 & \mu_{1} \mathbf{a}^{-} & \alpha_{1} \mathbf{k} \\
0 & 0 & 0 & 0 & 0 & -q \alpha_{1}^{-1} \mathbf{k} \mu_{1}^{-1} \mathbf{a}^{+}
\end{array}\right), \\
& \pi_{2}(T)=\left(\begin{array}{ccccccc}
1 & 0 & 0 & 0 & 0 & 0 & 0 \\
0 & \mu_{2} \mathbf{A}^{-} & \alpha_{2} \mathbf{K} & 0 & 0 & 0 & 0 \\
0 & -q^{3} \alpha_{2}^{-1} \mathbf{K} & \mu_{2}^{-1} \mathbf{A}^{+} & 0 & 0 & 0 & 0 \\
0 & 0 & 0 & 1 & 0 & 0 & 0 \\
0 & 0 & 0 & 0 & \mu_{2} \mathbf{A}^{-} & \alpha_{2} \mathbf{K} & 0 \\
0 & 0 & 0 & 0 & -q^{3} \alpha_{2}^{-1} \mathbf{K} & \mu_{2}^{-1} \mathbf{A}^{+} & 0 \\
0 & 0 & 0 & 0 & 0 & 0 & 1
\end{array}\right),
\end{aligned}
$$

where $\alpha_{i}, \mu_{i}$ are nonzero parameters and $[2]_{1}=q+q^{-1}$ as defined after (4). 
The Weyl group $W=\left\langle s_{1}, s_{2}\right\rangle$ is the Coxeter system with the relations

$$
s_{1}^{2}=s_{2}^{2}=1, \quad s_{2} s_{1} s_{2} s_{1} s_{2} s_{1}=s_{1} s_{2} s_{1} s_{2} s_{1} s_{2} .
$$

Thus we have the isomorphism $\pi_{212121} \simeq \pi_{121212}$. Let $\Phi$ be the corresponding intertwiner and denote by $F$ the checked intertwiner $\Phi^{\vee}$

$$
\begin{aligned}
& \pi_{212121} \Phi=\Phi \pi_{121212}, \quad \pi_{212121} F=F \pi_{212121}^{\prime}, \quad \pi_{212121}^{\prime}=P_{16} P_{25} P_{34} \pi_{121212} P_{16} P_{25} P_{34}, \\
& F=\Phi P_{16} P_{25} P_{34} \in \operatorname{End}\left(\mathcal{F}_{q^{3}} \otimes \mathcal{F}_{q} \otimes \mathcal{F}_{q^{3}} \otimes \mathcal{F}_{q} \otimes \mathcal{F}_{q^{3}} \otimes \mathcal{F}_{q}\right) .
\end{aligned}
$$

Define the matrix elements of $F$ and its parameter-free part $\mathcal{F}$ by

$$
\begin{aligned}
& F|i, j, k, l, m, n\rangle=\sum_{a, b, c, d, e, f} F_{i j k l m n}^{a b c d e f}|a, b, c, d, e, f\rangle, \\
& F_{i j k l m n}^{a b c d e f}=\mu_{1}^{3 c-3 k+d-l+3 e-3 m} \mu_{2}^{2 k-2 c+l-d+3 m-3 e+n-f} \mathcal{F}_{i j k l m n}^{a b c d e f} .
\end{aligned}
$$

Then the following properties are valid for $\mathcal{F}=\left(\mathcal{F}_{i j k l m n}^{a b c d e f}\right)$ :

$$
\begin{aligned}
& \mathcal{F}_{i j k l m n}^{a b c d e f} \in \mathbb{Z}[q], \\
& \mathcal{F}_{i j k l m n}^{a b c d e f}=0 \quad \text { unless } \quad\left(\begin{array}{c}
a+b+2 c+d+e \\
b+3 c+2 d+3 e+f
\end{array}\right)=\left(\begin{array}{c}
i+j+2 k+l+m \\
j+3 k+2 l+3 m+n
\end{array}\right), \\
& \mathcal{F}^{-1}=\mathcal{F}, \quad \mathcal{F}_{i j k l m n}^{a b c d e f}=\frac{\left(q^{6}\right)_{i}\left(q^{2}\right)_{j}\left(q^{6}\right)_{k}\left(q^{2}\right)_{l}\left(q^{6}\right)_{m}\left(q^{2}\right)_{n}}{\left(q^{6}\right)_{a}\left(q^{2}\right)_{b}\left(q^{6}\right)_{c}\left(q^{2}\right)_{d}\left(q^{6}\right)_{e}\left(q^{2}\right)_{f}} \mathcal{F}_{\text {abcdef }}^{i j k l m n} .
\end{aligned}
$$

Due to (29), $\mathcal{F}$ is the infinite direct sum of finite-dimensional matrices. The formula for $\left.\mathcal{F}_{i j k l m n}^{a b c d e f}\right|_{q=0}$ can be deduced by the ultradiscretization (tropical form) of [3, Theorem 3.1(c)]. Although a tedious algorithm can be formulated for calculating any given $\mathcal{F}_{i j k l m n}^{a b c d e f}$ by using (28), an explicit formula for it is yet to be constructed.

Example 3. The following is the list of all the nonzero $\mathcal{F}_{010101}^{a b c d e f}$ :

$$
\begin{aligned}
& \mathcal{F}_{010101}^{000200}=q^{4}\left(1-q^{2}\right)\left(1-q^{2}-q^{4}-q^{6}\right), \\
& \mathcal{F}_{001001}^{00101}=-q\left(1-q^{2}\right)\left(1-q^{2}-q^{4}+q^{8}+q^{10}\right), \\
& \mathcal{F}_{010101}^{010010}=-q\left(1-q^{2}\right)\left(1-q^{2}-q^{4}+q^{8}+q^{10}\right), \\
& \mathcal{F}_{010101}^{010101}=1-2 q^{2}+2 q^{6}+3 q^{8}-2 q^{12}-2 q^{14}-q^{16}, \\
& \mathcal{F}_{010101}^{020002}=q^{4}\left(-2+2 q^{6}+q^{8}+q^{10}\right), \\
& \mathcal{F}_{010101}^{100011}=-q^{3}\left(1-q^{2}\right)\left(1-q^{6}-q^{8}\right), \\
& \mathcal{F}_{010101}^{100102}=q\left(1-q^{2}-q^{4}-q^{6}+q^{10}+q^{12}+q^{14}\right), \\
& \mathcal{F}_{0101001}^{20000}=q^{4}, \\
& \mathcal{F}_{010101}^{11000}=q\left(1-q+q^{2}\right)\left(1+q+q^{2}\right)\left(1-q^{2}-q^{8}\right) .
\end{aligned}
$$

\section{Main theorem}

In this section we fix two reduced words $\mathbf{i}=\left(i_{1}, \ldots, i_{l}\right), \mathbf{j}=\left(j_{1}, \ldots, j_{l}\right)$ of the longest element $w_{0} \in W$. 


\subsection{Definitions of $\gamma_{B}^{A}$ and $\Phi_{B}^{A}$}

In the $U_{q}(\mathfrak{g})$ side, we defined the PBW bases $E_{\mathbf{i}}^{A}, E_{\mathbf{j}}^{B}$ of $U_{q}^{+}(\mathfrak{g})$ in Section 2.2. We define their transition coefficient $\gamma_{B}^{A}$ by

$$
E_{\mathbf{i}}^{A}=\sum_{B} \gamma_{B}^{A} E_{\mathbf{j}}^{B}
$$

While, in the $A_{q}(\mathfrak{g})$ side, we have the intertwiner $\Phi: \mathcal{F}_{q_{i_{1}}} \otimes \cdots \otimes \mathcal{F}_{q_{i_{l}}} \rightarrow \mathcal{F}_{q_{j_{1}}} \otimes \cdots \otimes \mathcal{F}_{q_{j_{l}}}$ satisfying

$$
\pi_{\mathbf{j}}(g) \circ \Phi=\Phi \circ \pi_{\mathbf{i}}(g) \quad \forall g \in A_{q}(\mathfrak{g}) .
$$

We take the parameters $\mu, \alpha$ in (18) to be 1 . This in particular means for rank 2 cases that $\mu_{i}, \alpha_{i}$ entering $\pi_{i}(T)$ in (19), (23) and (27) are all 1. The intertwiner $\Phi$ is normalized by $\Phi|0,0, \ldots, 0\rangle=|0,0, \ldots, 0\rangle$. Under these conditions a matrix element $\Phi_{B}^{A}$ of $\Phi$ is uniquely specified by

$$
\Phi|B\rangle=\sum_{A} \Phi_{B}^{A}|A\rangle
$$

where $A=\left(a_{1}, \ldots, a_{l}\right) \in\left(\mathbb{Z}_{\geq 0}\right)^{l}$ and $|A\rangle=\left|a_{1}\right\rangle \otimes \cdots \otimes\left|a_{l}\right\rangle \in \mathcal{F}_{q_{j_{1}}} \otimes \cdots \otimes \mathcal{F}_{q_{j_{l}}}$ and similarly for $|B\rangle \in \mathcal{F}_{q_{i_{1}}} \otimes \cdots \otimes \mathcal{F}_{q_{i_{l}}}$. Then our main result is

\section{Theorem 5 .}

$$
\gamma_{B}^{A}=\Phi_{B}^{A}
$$

For any pair $(\mathbf{i}, \mathbf{j})$, from $\mathbf{i}$ one can reach $\mathbf{j}$ by applying Coxeter relations. In view of the uniqueness of $\gamma$ and $\Phi$ and the fact that the braid group action $T_{i}$ is an algebra homomorphism, the proof of this theorem reduces to establishing the same equality for all $\mathfrak{g}$ of rank 2 . This will be done in the rest of this section.

\subsection{Proof of Theorem 5 for rank 2 cases}

In the rank 2 cases, there are two reduced expressions $s_{i_{1}} \cdots s_{i_{l}}$ for the longest element of the Weyl group. Denote the associated sequences $\mathbf{i}=\left(i_{1}, \ldots, i_{l}\right)$ by $\mathbf{1}, \mathbf{2}$ and set $\mathbf{1}^{\prime}=\mathbf{2}, \mathbf{2}^{\prime}=\mathbf{1}$. Concretely, we take them as

$$
\begin{array}{llll}
A_{2}: & \mathbf{1}=(1,2,1), & \mathbf{2}=(2,1,2), & \left(q_{1}, q_{2}\right)=(q, q), \\
C_{2}: \quad \mathbf{1}=(1,2,1,2), & \mathbf{2}=(2,1,2,1), & \left(q_{1}, q_{2}\right)=\left(q, q^{2}\right), \\
G_{2}: & \mathbf{1}=(1,2,1,2,1,2), & \mathbf{2}=(2,1,2,1,2,1), & \left(q_{1}, q_{2}\right)=\left(q, q^{3}\right),
\end{array}
$$

where $q_{i}$ defined after (4) is also recalled. In order to simplify the formulae in Section 5.3, we use the PBW bases and the Fock states in yet another normalization as follows:

$$
\begin{aligned}
\tilde{E}_{\mathbf{i}}^{A} & :=\left(\left[a_{1}\right]_{i_{1}} ! \cdots\left[a_{l}\right]_{i_{l}} !\right) E_{\mathbf{i}}^{A}=e_{\beta_{1}}^{a_{1}} \cdots e_{\beta_{l}}^{a_{l}}, \\
|A\rangle\rangle: & :=d_{i_{1}, a_{1}} \cdots d_{i_{l}, a_{l}}|A\rangle, \quad d_{i, a}=q_{i}^{-a(a-1) / 2} \lambda_{i}^{a}, \quad \lambda_{i}=\left(1-q_{i}^{2}\right)^{-1},
\end{aligned}
$$

where $A=\left(a_{1}, \ldots, a_{l}\right)$. See after (4) for the symbol $[a]_{i} ! . e_{\beta_{r}}$ is defined in (5). Accordingly we introduce the matrix elements $\tilde{\gamma}_{B}^{A}$ and $\tilde{\Phi}_{B}^{A}$ by

$$
\left.\left.\tilde{E}_{\mathbf{i}}^{A}=\sum_{B} \tilde{\gamma}_{B}^{A} \tilde{E}_{\mathbf{i}^{\prime}}^{B}, \quad \Phi|B\rangle\right\rangle=\sum_{A} \tilde{\Phi}_{B}^{A}|A\rangle\right\rangle, \quad \mathbf{i}=\mathbf{1}, \mathbf{2} .
$$


It follows that $\gamma_{B}^{A}=\tilde{\gamma}_{B}^{A} \prod_{k=1}^{l}\left(\left[b_{k}\right]_{i_{k}} ! /\left[a_{k}\right]_{i_{k}} !\right)$ and $\Phi_{B}^{A}=\tilde{\Phi}_{B}^{A} \prod_{k=1}^{l}\left(d_{i_{k}, a_{k}} / d_{i_{k}, b_{k}}\right)$ for $B=\left(b_{1}, \ldots, b_{l}\right)$.

On the other hand, we know $\Phi_{B}^{A}=\Phi_{A}^{B} \prod_{k=1}^{l}\left(\left(q_{i_{k}}^{2}\right)_{b_{k}} /\left(q_{i_{k}}^{2}\right)_{a_{k}}\right)$ from (21), (25) and (30). Due to the identity $\left(q_{i}^{2}\right)_{m} d_{i, m}=[m]_{i}$ !, the assertion $\gamma_{B}^{A}=\Phi_{B}^{A}$ of Theorem 5 is equivalent to

$$
\tilde{\gamma}_{B}^{A}=\tilde{\Phi}_{A}^{B}
$$

Let $\rho_{\mathbf{i}}(x)=\left(\rho_{\mathbf{i}}(x)_{A B}\right)$ be the matrix for the left multiplication of $x \in U_{q}^{+}(\mathfrak{g})$ :

$$
x \cdot \tilde{E}_{\mathbf{i}}^{A}=\sum_{B} \tilde{E}_{\mathbf{i}}^{B} \rho_{\mathbf{i}}(x)_{B A} .
$$

Let further $\pi_{\mathbf{i}}(g)=\left(\pi_{\mathbf{i}}(g)_{A B}\right)$ be the representation matrix of $g \in A_{q}(\mathfrak{g})$ :

$$
\left.\left.\pi_{\mathbf{i}}(g)|A\rangle\right\rangle=\sum_{B}|B\rangle\right\rangle \pi_{\mathbf{i}}(g)_{B A} .
$$

The following element in the right quotient $\operatorname{ring} A_{q}(\mathfrak{g})_{\mathcal{S}}$ will play a key role in our proof.

$$
\xi_{i}=\lambda_{i}\left(\sigma_{i} e_{i}\right) / \sigma_{i}, \quad i=1,2 .
$$

See Definition 1 for $\sigma_{i}$ and (39), (41), (42) for the concrete forms in rank 2 cases. In Section 5.3 we will check the following statement case by case.

Proposition 2. For $\mathfrak{g}$ of rank $2, \pi_{\mathbf{i}}\left(\sigma_{i}\right)$ is invertible and the following equality is valid:

$$
\rho_{\mathbf{i}}\left(e_{i}\right)_{A B}=\pi_{\mathbf{i}}\left(\xi_{i}\right)_{A B}, \quad i=1,2,
$$

where the right-hand side means $\lambda_{i} \pi_{\mathbf{i}}\left(\sigma_{i} e_{i}\right) \pi_{\mathbf{i}}\left(\sigma_{i}\right)^{-1}$.

Proof of Theorem 5 for rank 2 case. We write the both sides of (37) as $M_{A B}^{i}$ and the one for $\mathbf{i}^{\prime}$ instead of $\mathbf{i}$ as $M_{A B}^{\prime i}$. From

$$
\sum_{B, C} \tilde{E}_{\mathbf{i}^{\prime}}^{C} M_{C B}^{\prime i} \tilde{\gamma}_{B}^{A}=e_{i} \sum_{B} \tilde{E}_{\mathbf{i}^{\prime}}^{B} \tilde{\gamma}_{B}^{A}=e_{i} \tilde{E}_{\mathbf{i}}^{A}=\sum_{B} \tilde{E}_{\mathbf{i}}^{B} M_{B A}^{i}=\sum_{B, C} \tilde{E}_{\mathbf{i}^{\prime}}^{C} \tilde{\gamma}_{C}^{B} M_{B A}^{i}
$$

we have $\sum_{B} M_{C B}^{\prime i} \tilde{\gamma}_{B}^{A}=\sum_{B} \tilde{\gamma}_{C}^{B} M_{B A}^{i}$. On the other hand, the action of the two sides of (31) with $g=\xi_{i}$ and $\mathbf{j}=\mathbf{i}^{\prime}$ are calculated as

$$
\left.\left.\left.\pi_{\mathbf{i}^{\prime}}\left(\xi_{i}\right) \circ \Phi|A\rangle\right\rangle=\pi_{\mathbf{i}^{\prime}}\left(\xi_{i}\right) \sum_{B}|B\rangle\right\rangle \tilde{\Phi}_{A}^{B}=\sum_{B, C}|C\rangle\right\rangle M_{C B}^{\prime i} \tilde{\Phi}_{A}^{B}
$$

and

$$
\left.\left.\left.\Phi \circ \pi_{\mathbf{i}}\left(\xi_{i}\right)|A\rangle\right\rangle=\Phi \sum_{B}|B\rangle\right\rangle M_{B A}^{i}=\sum_{B, C}|C\rangle\right\rangle \tilde{\Phi}_{B}^{C} M_{B A}^{i} .
$$

Hence $\sum_{B} M_{C B}^{\prime i} \tilde{\Phi}_{A}^{B}=\sum_{B} \tilde{\Phi}_{B}^{C} M_{B A}^{i}$. Thus $\tilde{\gamma}_{B}^{A}$ and $\tilde{\Phi}_{A}^{B}$ satisfy the same relation. Moreover the maps $\pi_{\mathbf{i}}$ and $\rho_{\mathbf{i}}$ are both homomorphism, i.e., $\pi_{\mathbf{i}}(g h)=\pi_{\mathbf{i}}(g) \pi_{\mathbf{i}}(h)$ and $\rho_{\mathbf{i}}(x y)=\rho_{\mathbf{i}}(x) \rho_{\mathbf{i}}(y)$. We know that $\Phi$ is the intertwiner of the irreducible $A_{q}(\mathfrak{g})$ modules and (33) obviously holds as $1=1$ at $A=B=(0, \ldots, 0)$. Thus it is valid for arbitrary $A$ and $B$.

Conjecture 1. The equality (37) is valid for any $\mathfrak{g}$. 


\subsection{Explicit formulae for rank 2 cases: Proof of Proposition 2}

Here we present the explicit formulae of (34) with $x=e_{i}$ and (35) with $g=\sigma_{i}, \sigma_{i} e_{i}$ that allow one to check Proposition 2. We use the notation $\langle i\rangle=q^{i}-q^{-i}$. In each case, there are two i-sequences, $\mathbf{1}$ and $\mathbf{2}=\mathbf{1}^{\prime}$ corresponding to the two reduced words. Let $\chi$ be the anti-algebra involution such that $\chi\left(e_{i}\right)=e_{i}$. Then the relation $\chi\left(\tilde{E}_{\mathbf{i}}^{A}\right)=\tilde{E}_{\mathbf{i}^{\prime}}^{\bar{A}}$ holds, where $\bar{A}=\left(a_{l}, \ldots, a_{2}, a_{1}\right)$ denotes the reversal of $A=\left(a_{1}, a_{2}, \ldots, a_{l}\right)$. Applying $\chi$ to (34) with $x=e_{i}$ yields the right multiplication formula $\tilde{E}_{\mathbf{i}^{\prime}}^{\bar{A}} \cdot e_{i}=\sum_{B} \tilde{E}_{\mathbf{i}^{\prime}}^{\bar{B}} \rho_{\mathbf{i}}\left(e_{i}\right)_{B A}$ for $\mathbf{i}^{\prime}$-sequence. In view of this fact, we shall present the left and right multiplication formulae for $\mathbf{i}=\mathbf{2}$ only.

As for (35) with $g=\xi_{i}$ in (36), explicit formulae for $\sigma_{i}, \sigma_{i} e_{i} \in A_{q}(\mathfrak{g})$ and their image by the both representations $\pi_{\mathbf{1}}$ and $\pi_{\mathbf{2}}$ will be given. We include an exposition on how to use these data to check (37) along the simplest $A_{2}$ case. The $C_{2}$ and $G_{2}$ cases are similar.

\subsection{1 $\quad A_{2}$ case}

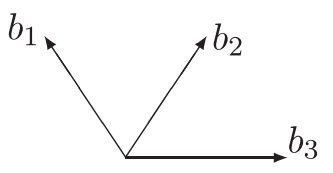

The $q$-Serre relations are

$$
e_{1}^{2} e_{2}-[2]_{1} e_{1} e_{2} e_{1}+e_{2} e_{1}^{2}=0, \quad e_{2}^{2} e_{1}-[2]_{1} e_{2} e_{1} e_{2}+e_{1} e_{2}^{2}=0,
$$

where $[m]_{1}=\langle m\rangle /\langle 1\rangle$. Let $b_{1}, b_{2}, b_{3}$ be the generator for positive roots: $b_{1}=e_{2}, b_{2}=e_{1} e_{2}-q e_{2} e_{1}$ and $b_{3}=e_{1}$. In the notation of Section 2.2, they are the root vectors $b_{i}=e_{\beta_{i}}$ associated with the reduced expression $w_{0}=s_{2} s_{1} s_{2}$ for $\mathbf{2}=(2,1,2)$. The corresponding positive roots are $\left(\beta_{1}, \beta_{2}, \beta_{3}\right)=\left(\alpha_{2}, \alpha_{1}+\alpha_{2}, \alpha_{1}\right)$. In particular, $b_{2}=T_{2}\left(e_{1}\right)$. Their commutation relations are $b_{2} b_{1}=q^{-1} b_{1} b_{2}, b_{3} b_{1}=b_{2}+q b_{1} b_{3}, b_{3} b_{2}=q^{-1} b_{2} b_{3}$.

Lemma 2. For $\tilde{E}_{\mathbf{2}}^{a, b, c}=b_{1}^{a} b_{2}^{b} b_{3}^{c}$, we have

$$
\begin{aligned}
& \tilde{E}_{\mathbf{2}}^{a, b, c} \cdot e_{1}=\tilde{E}_{\mathbf{2}}^{a, b, c+1}, \\
& \tilde{E}_{\mathbf{2}}^{a, b, c} \cdot e_{2}=q^{c-b} \tilde{E}_{\mathbf{2}}^{a+1, b, c}+[c]_{1} \tilde{E}_{\mathbf{2}}^{a, b+1, c-1}, \\
& e_{1} \cdot \tilde{E}_{\mathbf{2}}^{a, b, c}=q^{a-b} \tilde{E}_{\mathbf{2}}^{a, b, c+1}+[a]_{1} \tilde{E}_{\mathbf{2}}^{a-1, b+1, c}, \\
& e_{2} \cdot \tilde{E}_{\mathbf{2}}^{a, b, c}=\tilde{E}_{\mathbf{2}}^{a+1, b, c} .
\end{aligned}
$$

Proof. By induction, we have

$$
\begin{array}{ll}
b_{3} b_{1}^{n}=q^{n} b_{1}^{n} b_{3}+[n]_{1} b_{1}^{n-1} b_{2}, & b_{3} b_{2}^{n}=q^{-n} b_{2}^{n} b_{3}, \\
b_{3}^{n} b_{1}=q^{n} b_{1} b_{3}^{n}+[n]_{1} b_{2} b_{3}^{n-1}, & b_{2}^{n} b_{1}=q^{-n} b_{1} b_{2}^{n} .
\end{array}
$$

The lemma is a direct consequence of these formulae.

Set $\tilde{E}_{\mathbf{1}}^{a, b, c}=\chi\left(\tilde{E}_{\mathbf{2}}^{c, b, a}\right)=\chi\left(b_{3}^{a}\right) \chi\left(b_{2}^{b}\right) \chi\left(b_{1}^{c}\right)=b_{3}^{a} b_{2}^{\prime b} b_{1}^{c}$, where $b_{2}^{\prime}:=\chi\left(b_{2}\right)=e_{2} e_{1}-q e_{1} e_{2}$. By applying $\chi$ to the first two relations in Lemma 2 , we get

$$
e_{1} \cdot \tilde{E}_{\mathbf{1}}^{a, b, c}=\tilde{E}_{\mathbf{1}}^{a+1, b, c}, \quad e_{2} \cdot \tilde{E}_{\mathbf{1}}^{a, b, c}=q^{a-b} E_{\mathbf{1}}^{a, b, c+1}+[a]_{1} \tilde{E}_{\mathbf{1}}^{a-1, b+1, c} .
$$

Thus we find $\rho_{\mathbf{i}^{\prime}}\left(e_{i}\right)=\rho_{\mathbf{i}}\left(e_{3-i}\right)$. This property is only valid for $A_{2}$ and not in $C_{2}$ and $G_{2}$.

Let us turn to the representations $\pi_{\mathbf{i}}$ of $A_{q}\left(A_{2}\right)$. The elements $\sigma_{i}$ in Definition 1 and $\sigma_{i} e_{i}$ are given by

$$
\sigma_{1}=t_{13}, \quad \sigma_{2}=t_{12} t_{23}-q t_{22} t_{13}, \quad \sigma_{1} e_{1}=t_{23}, \quad \sigma_{2} e_{2}=t_{12} t_{33}-q t_{32} t_{13} .
$$

See the exposition at the end of Section 3.3.1 and the remark after Lemma 1. 
From (14) and (19) with $\alpha_{i}=\mu_{i}=1$, we find

$$
\pi_{\mathbf{1}}\left(\sigma_{1}\right)=\mathbf{k}_{1} \mathbf{k}_{2}, \quad \pi_{\mathbf{1}}\left(\sigma_{1} e_{1}\right)=\mathbf{a}_{1}^{+} \mathbf{k}_{2}, \quad \pi_{\mathbf{1}}\left(\sigma_{2}\right)=\mathbf{k}_{2} \mathbf{k}_{3}, \quad \pi_{\mathbf{1}}\left(\sigma_{2} e_{2}\right)=\mathbf{a}_{1}^{-} \mathbf{a}_{2}^{+} \mathbf{k}_{3}+\mathbf{k}_{1} \mathbf{a}_{3}^{+},
$$

where the notation like $\mathbf{k}_{1} \mathbf{a}_{3}^{+}=\mathbf{k} \otimes 1 \otimes \mathbf{a}^{+}$has been used. Since $\mathbf{k} \in \operatorname{End}\left(\mathcal{F}_{q}\right)$ is invertible, so is $\pi_{\mathbf{i}}\left(\sigma_{i}\right)$ and we may write

$$
\pi_{\mathbf{1}}\left(\xi_{1}\right)=\lambda_{1} \mathbf{a}_{1}^{+} \mathbf{k}_{1}^{-1}, \quad \pi_{\mathbf{1}}\left(\xi_{2}\right)=\lambda_{2}\left(\mathbf{a}_{1}^{-} \mathbf{a}_{2}^{+} \mathbf{k}_{2}^{-1}+\mathbf{k}_{1} \mathbf{k}_{2}^{-1} \mathbf{a}_{3}^{+} \mathbf{k}_{3}^{-1}\right),
$$

where $\lambda_{1}=\lambda_{2}=\left(1-q^{2}\right)^{-1}$. The action of each component on the ket vector $\left.|m\rangle\right\rangle:=d_{i, m}|m\rangle \in$ $\mathcal{F}_{q_{i}}$ (cf. (32)) takes the form

$$
\left.\left.\left.\left.\left.\left.\mathbf{a}^{+}|m\rangle\right\rangle=\lambda_{i}^{-1} q_{i}^{m}|m+1\rangle\right\rangle, \quad \mathbf{a}^{-}|m\rangle\right\rangle=[m]_{i}|m-1\rangle\right\rangle, \quad \mathbf{k}|m\rangle\right\rangle=q_{i}^{m}|m\rangle\right\rangle,
$$

due to (17). (The formula (40) is valid also for $C_{2}$ and $G_{2}$ provided that $\mathbf{a}^{+}, \mathbf{a}^{-}, \mathbf{k}$ are interpreted as $\mathbf{A}^{+}, \mathbf{A}^{-}, \mathbf{K}$ for $i=2$.) Thus one has

$$
\left.\left.\left.\left.\left.\pi_{\mathbf{1}}\left(\xi_{1}\right)|a, b, c\rangle\right\rangle=|a+1, b, c\rangle\right\rangle, \quad \pi_{\mathbf{1}}\left(\xi_{2}\right)|a, b, c\rangle\right\rangle=[a]_{1}|a-1, b+1, c\rangle\right\rangle+q^{a-b}|a, b, c+1\rangle\right\rangle .
$$

This agrees with (38) thereby proving (37) for $\mathbf{i}=\mathbf{1}$. The other case $\mathbf{i}=\mathbf{2}$ also holds due to the symmetry $\pi_{\mathbf{2}}\left(\xi_{i}\right)=\pi_{\mathbf{1}}\left(\xi_{3-i}\right)$. Thus Proposition 2 is established for $A_{2}$.

In terms of the checked intertwiner $\mathcal{R}$ in Section 4.2 , Theorem 5 implies

$$
E_{\mathbf{i}}^{a, b, c}=\sum_{i, j, k} \mathcal{R}_{i j k}^{a b c} E_{\mathbf{i}^{\prime}}^{k, j, i}
$$

This is valid either for $\mathbf{i}=\mathbf{1}$ or $\mathbf{2}$ thanks to the middle property in (21). This relation connecting the PBW bases with the solution of the tetrahedron equation is due to [20].

\subsection{2 $\quad C_{2}$ case}

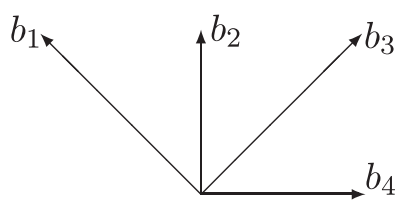

The $q$-Serre relations are

$$
e_{1}^{3} e_{2}-[3]_{1} e_{1}^{2} e_{2} e_{1}+[3]_{1} e_{1} e_{2} e_{1}^{2}-e_{2} e_{1}^{3}=0, \quad e_{2}^{2} e_{1}-[2]_{2} e_{2} e_{1} e_{2}+e_{1} e_{2}^{2}=0,
$$

where $[m]_{1}=\langle m\rangle /\langle 1\rangle$ and $[m]_{2}=\langle 2 m\rangle /\langle 2\rangle$.

Let $b_{1}, \ldots, b_{4}$ be the generator for positive roots: $b_{1}=e_{2}, b_{2}=e_{1} e_{2}-q^{2} e_{2} e_{1}, b_{3}=\frac{1}{[2]_{1}}\left(e_{1} b_{2}-\right.$ $\left.b_{2} e_{1}\right)$ and $b_{4}=e_{1}$. Their commutation relations are $b_{2} b_{1}=q^{-2} b_{1} b_{2}, b_{3} b_{1}=-q^{-1}\langle 1\rangle[2]_{1}^{-1} b_{2}^{2}+b_{1} b_{3}$, $b_{4} b_{1}=b_{2}+q^{2} b_{1} b_{4}, b_{3} b_{2}=q^{-2} b_{2} b_{3}, b_{4} b_{2}=[2]_{1} b_{3}+b_{2} b_{4}, b_{4} b_{3}=q^{-2} b_{3} b_{4}$.

Lemma 3. For $\tilde{E}_{\mathbf{2}}^{a, b, c, d}=b_{1}^{a} b_{2}^{b} b_{3}^{c} b_{4}^{d}$, we have

$$
\begin{aligned}
\tilde{E}_{\mathbf{2}}^{a, b, c, d} \cdot e_{1}= & \tilde{E}_{\mathbf{2}}^{a, b, c, d+1}, \\
\tilde{E}_{\mathbf{2}}^{a, b, c, d} \cdot e_{2}= & {[d]_{1} q^{d-2 c-1} \tilde{E}_{\mathbf{2}}^{a, b+1, c, d-1}+q^{2(d-b)} \tilde{E}_{\mathbf{2}}^{a+1, b, c, d} } \\
& -\langle 1\rangle q^{2 d-2 c+1}[c]_{2}[2]_{1}^{-1} \tilde{E}_{\mathbf{2}}^{a, b+2, c-1, d}+[d-1]_{1}[d]_{1} \tilde{E}_{\mathbf{2}}^{a, b, c+1, d-2}, \\
e_{1} \cdot \tilde{E}_{\mathbf{2}}^{a, b, c, d}= & {[2]_{1}[b]_{1} q^{2 a-b+1} \tilde{E}_{\mathbf{2}}^{a, b-1, c+1, d}+q^{2 a-2 c} \tilde{E}_{\mathbf{2}}^{a, b, c, d+1}+[a]_{2} \tilde{E}_{\mathbf{2}}^{a-1, b+1, c, d}, } \\
e_{2} \cdot \tilde{E}_{\mathbf{2}}^{a, b, c, d}= & \tilde{E}_{\mathbf{2}}^{a+1, b, c, d} .
\end{aligned}
$$


Proof. By induction, we have

$$
\begin{aligned}
& b_{4} b_{1}^{n}=b_{1}^{n} b_{4} q^{2 n}+[n]_{2} b_{1}^{n-1} b_{2}, \quad b_{4} b_{2}^{n}=[2]_{1}[n]_{1} b_{2}^{n-1} b_{3} q^{-n+1}+b_{2}^{n} b_{4}, \\
& b_{4} b_{3}^{n}=q^{-2 n} b_{3}^{n} b_{4}, \quad b_{4}^{n} b_{1}=[n]_{1} b_{2} b_{4}^{n-1} q^{n-1}+b_{1} b_{4}^{n} q^{2 n}+[n-1]_{1}[n]_{1} b_{3} b_{4}^{n-2}, \\
& b_{3}^{n} b_{1}=-q^{1-2 n}\langle 1\rangle[n]_{2}[2]_{1}^{-1} b_{2}^{2} b_{3}^{n-1}+b_{1} b_{3}^{n}, \quad b_{3}^{n} b_{2}=q^{-2 n} b_{2} b_{3}^{n}, \quad b_{2}^{n} b_{1}=q^{-2 n} b_{1} b_{2}^{n} .
\end{aligned}
$$

The lemma is a direct consequence of these formulae.

Set $\tilde{E}_{\mathbf{1}}^{a, b, c, d}=\chi\left(\tilde{E}_{\mathbf{2}}^{d, c, b, a}\right)$. The left multiplication formula for this basis is deduced from the above lemma by applying $\chi$. One can adjust the definition of $E_{\mathbf{i}}^{A}$ in (6) with that in [24] by setting $v=q^{-1}$.

Let us turn to the representations $\pi_{\mathbf{i}}$ of $A_{q}\left(C_{2}\right)$. The elements $\sigma_{i}$ in Definition 1 and $\sigma_{i} e_{i}$ are given by

$$
\sigma_{1}=t_{14}, \quad \sigma_{2}=t_{13} t_{24}-q t_{23} t_{14}, \quad \sigma_{1} e_{1}=t_{24}, \quad \sigma_{2} e_{2}=t_{13} t_{34}-q t_{33} t_{14} .
$$

From (14) and (23) with $\alpha_{i}=\mu_{i}=1$, we have

$$
\begin{aligned}
& \pi_{\mathbf{1}}\left(\sigma_{1}\right)=-\mathbf{k}_{1} \mathbf{K}_{2} \mathbf{k}_{3}, \\
& \pi_{\mathbf{1}}\left(\sigma_{1} e_{1}\right)=-\mathbf{a}_{1}^{+} \mathbf{K}_{2} \mathbf{k}_{3}, \\
& \pi_{\mathbf{1}}\left(\sigma_{2}\right)=-\mathbf{K}_{2} \mathbf{k}_{3}{ }^{2} \mathbf{K}_{4}, \\
& \left.\pi_{\mathbf{1}}\left(\sigma_{2} e_{2}\right)=-\mathbf{a}_{1}^{-2} \mathbf{A}_{2}^{+} \mathbf{k}_{3}{ }^{2} \mathbf{K}_{4}-[2]\right]_{1} \mathbf{a}_{1}^{-} \mathbf{k}_{1} \mathbf{a}_{3}^{+} \mathbf{k}_{3} \mathbf{K}_{4}-\mathbf{k}_{1}{ }^{2} \mathbf{A}_{2}^{-} \mathbf{a}_{3}^{+2} \mathbf{K}_{4}-\mathbf{A}_{4}^{+} \mathbf{k}_{1}{ }^{2} \mathbf{K}_{2}, \\
& \lambda_{1}^{-1} \pi_{\mathbf{1}}\left(\xi_{1}\right)=\mathbf{a}_{1}^{+} \mathbf{k}_{1}{ }^{-1} \\
& \lambda_{2}^{-1} \pi_{\mathbf{1}}\left(\xi_{2}\right)=\mathbf{a}_{1}^{-2} \mathbf{A}_{2}^{+} \mathbf{K}_{2}{ }^{-1}+\mathbf{k}_{1}{ }^{2} \mathbf{A}_{2}^{-} \mathbf{K}_{2}{ }^{-1} \mathbf{a}_{3}^{+}{ }^{2} \mathbf{k}_{3}{ }^{-2} \\
& \quad \quad+[2]_{1} \mathbf{a}_{1}^{-} \mathbf{k}_{1} \mathbf{K}_{2}{ }^{-1} \mathbf{a}_{3}^{+} \mathbf{k}_{3}{ }^{-1}+\mathbf{k}_{1}{ }^{2} \mathbf{k}_{3}{ }^{-2} \mathbf{A}_{4}^{+} \mathbf{K}_{4}{ }^{-1} \\
& \pi_{\mathbf{2}}\left(\sigma_{1}\right)=-\mathbf{k}_{2} \mathbf{K}_{3} \mathbf{k}_{4}, \\
& \pi_{\mathbf{2}}\left(\sigma_{1} e_{1}\right)=-\mathbf{K}_{1} \mathbf{k}_{2} \mathbf{a}_{4}^{+}-\mathbf{K}_{1} \mathbf{a}_{2}^{-} \mathbf{A}_{3}^{+} \mathbf{k}_{4}-\mathbf{A}_{1}^{-} \mathbf{a}_{2}^{+} \mathbf{K}_{3} \mathbf{k}_{4}, \\
& \pi_{\mathbf{2}}\left(\sigma_{2}\right)=-\mathbf{K}_{1} \mathbf{k}_{2}^{2} \mathbf{K}_{3}, \\
& \pi_{\mathbf{2}}\left(\sigma_{2} e_{2}\right)=-\mathbf{A}_{1}^{+} \mathbf{k}_{2}^{2} \mathbf{K}_{3}, \\
& \lambda_{1}^{-1} \pi_{\mathbf{2}}\left(\xi_{1}\right)=\mathbf{A}_{1}^{-} \mathbf{a}_{2}^{+} \mathbf{k}_{2}^{-1}+\mathbf{K}_{1} \mathbf{a}_{2}^{-} \mathbf{k}_{2}^{-1} \mathbf{A}_{3}^{+} \mathbf{K}_{3}^{-1}+\mathbf{K}_{1} \mathbf{K}_{3}^{-1} \mathbf{a}_{4}^{+} \mathbf{k}_{4}^{-1} \\
& \lambda_{2}^{-1} \pi_{\mathbf{2}}\left(\xi_{2}\right)=\mathbf{A}_{1}^{+} \mathbf{K}_{1}{ }^{-1} .
\end{aligned}
$$

We find that $\pi_{\mathbf{i}}\left(\sigma_{i}\right)$ is invertible. Comparing these formulae with Lemma 3 by using (40), the equality (37) is directly checked. Thus Proposition 2 is established for $C_{2}$.

In terms of the checked intertwiner $\mathcal{K}$ in Section 4.3, Theorem 5 implies

$$
E_{\mathbf{2}}^{a, b, c, d}=\sum_{i, j, k, l} \mathcal{K}_{i j k l}^{a b c d} E_{\mathbf{1}}^{l, k, j, i}
$$

Thus the solution to the $3 \mathrm{D}$ reflection equation [13] is identified with the transition coefficient of the PBW bases for $U_{q}^{+}\left(C_{2}\right)$.

\section{$5.4 G_{2}$ case}

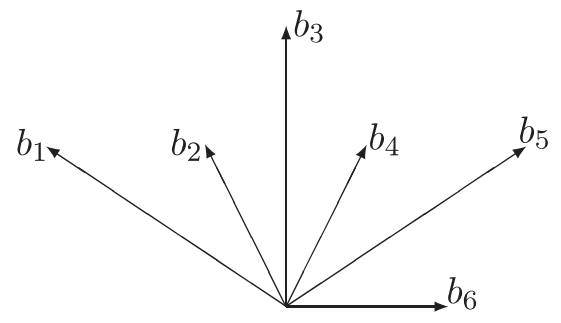


The $q$-Serre relations are

$$
\begin{aligned}
& e_{1}^{4} e_{2}-[4]_{1} e_{1}^{3} e_{2} e_{1}+[4]_{1}[3]_{1} /[2]_{1}^{-1} e_{1}^{2} e_{2} e_{1}^{2}-[4]_{1} e_{1} e_{2} e_{1}^{3}+e_{2} e_{1}^{4}=0, \\
& e_{2}^{2} e_{1}-[2]_{2} e_{2} e_{1} e_{2}+e_{1} e_{2}^{2}=0
\end{aligned}
$$

where we remind that $[m]_{1}=\langle m\rangle /\langle 1\rangle$ and $[m]_{2}=\langle 3 m\rangle /\langle 3\rangle$.

Let $b_{1}, \ldots, b_{6}$ be the generator for positive roots: $b_{1}=e_{2}, b_{2}=e_{1} e_{2}-q^{3} e_{2} e_{1}, b_{4}=\frac{1}{[2]_{1}}\left(e_{1} b_{2}-\right.$ $\left.q b_{2} e_{1}\right), b_{5}=\frac{1}{[3]_{1}}\left(e_{1} b_{4}-q^{-1} b_{4} e_{1}\right), b_{3}=\frac{1}{[3]_{1}}\left(b_{4} b_{2}-q^{-1} b_{2} b_{4}\right)$ and $b_{6}=e_{1}$. Their commutation relations are as follows: $b_{2} b_{1}=b_{1} b_{2} q^{-3}, b_{3} b_{1}=\langle 1\rangle^{2} b_{2}^{3} q^{-3}[3]_{1}^{-1}+b_{1} b_{3} q^{-3}, b_{4} b_{1}=b_{1} b_{4}-b_{2}^{2}\langle 1\rangle q^{-1}$, $b_{5} b_{1}=b_{1} b_{5} q^{3}-b_{2} b_{4}\langle 1\rangle q^{-1}-\left(q^{4}+q^{2}-1\right) b_{3} q^{-3}, b_{6} b_{1}=b_{1} b_{6} q^{3}+b_{2}, b_{3} b_{2}=b_{2} b_{3} q^{-3}, b_{4} b_{2}=b_{2} b_{4} q^{-1}+$ $b_{3}[3]_{1}, b_{5} b_{2}=b_{2} b_{5}-b_{4}^{2}\langle 1\rangle q^{-1}, b_{6} b_{2}=q b_{2} b_{6}+b_{4}[2]_{1}, b_{4} b_{3}=b_{3} b_{4} q^{-3}, b_{5} b_{3}=\langle 1\rangle^{2} b_{4}^{3} q^{-3}[3]_{1}^{-1}+$ $b_{3} b_{5} q^{-3}, b_{6} b_{3}=b_{3} b_{6}-b_{4}^{2}\langle 1\rangle q^{-1}, b_{5} b_{4}=b_{4} b_{5} q^{-3}, b_{6} b_{4}=[3]_{1} b_{5}+b_{4} b_{6} q^{-1}, b_{6} b_{5}=b_{5} b_{6} q^{-3}$.

Lemma 4. For $\tilde{E}_{\mathbf{2}}^{a, b, c, d, e, f}=b_{1}^{a} b_{2}^{b} \cdots b_{6}^{f}$, we have

$$
\begin{aligned}
\tilde{E}_{\mathbf{2}}^{a, b, c, d, e, f} \cdot e_{1}= & \tilde{E}_{\mathbf{2}}^{a, b, c, d, e, f+1}, \\
\tilde{E}_{\mathbf{2}}^{a, b, c, d, e, f} \cdot e_{2}= & -\langle 1\rangle[e]_{2} q^{-3 c-d+3 f-1} \tilde{E}_{\mathbf{2}}^{a, b+1, c, d+1, e-1, f} \\
& +\langle 1\rangle^{2}[e-1]_{2}[e]_{2}[3]_{1}^{-1} q^{-3 e+3 f+3} \tilde{E}_{\mathbf{2}}^{a, b, c, d+3, e-2, f} \\
& -\langle 3\rangle[d-1]_{1}[d]_{1} q^{-3 c-2 d+3 e+3 f+1} \tilde{E}_{\mathbf{2}}^{a, b+1, c+1, d-2, e, f} \\
& -\langle 1\rangle[d]_{1} q^{-6 c-d+3(e+f)} \tilde{E}_{\mathbf{2}}^{a, b+2, c, d-1, e, f}+[f-1]_{1}[f]_{1} q^{-3 e+f-2} \tilde{E}_{\mathbf{2}}^{a, b, c, d+1, e, f-2} \\
& +[3]_{1}[d]_{1}[f]_{1} q^{2 f-2 d} \tilde{E}_{\mathbf{2}}^{a, b, c+1, d-1, e, f-1}+[f]_{1} q^{-3 c-d+2 f-2} \tilde{E}_{\mathbf{2}}^{a, b+1, c, d, e, f-1} \\
& +q^{-3(b+c-e-f)} \tilde{E}_{\mathbf{2}}^{a+1, b, c, d, e, f}+\langle 1\rangle^{2}[c]_{2}[3]_{1}^{-1} q^{3(-2 c+e+f+1)} \tilde{E}_{\mathbf{2}}^{a, b+3, c-1, d, e, f} \\
& -\langle 3\rangle[d-2]_{1}[d-1]_{1}[d]_{1} q^{3(-d+e+f+2)} \tilde{E}_{\mathbf{2}}^{a, b, c+2, d-3, e, f} \\
& -\langle 1\rangle[e]_{2}[f]_{1} q^{-3 e+2 f} \tilde{E}_{\mathbf{2}}^{a, b, c, d+2, e-1, f-1} \\
& -[e]_{2} q^{-3 d+3 f}\left(q^{2 d+1}[3]_{1}-[2]_{2}\right) \tilde{E}_{\mathbf{2}}^{a, b, c+1, d, e-1, f} \\
& +[f-2]_{1}[f-1]_{1}[f]_{1} \tilde{E}_{\mathbf{2}}^{a, b, c, d, e+1, f-3}, \\
e_{1} \cdot \tilde{E}_{\mathbf{2}}^{a, b, c, d, e, f}= & -\langle 1\rangle[c]_{2} q^{3 a+b-3 c+2} \tilde{E}_{\mathbf{2}}^{a, b, c-1, d+2, e, f}+[3]_{1}[b-1]_{1}[b]_{1} q^{3 a-b+2} \tilde{E}_{\mathbf{2}}^{a, b-2, c+1, d, e, f} \\
& +[3]_{1}[d]_{1} q^{3 a+b-2 d+2} \tilde{E}_{\mathbf{2}}^{a, b, c, d-1, e+1, f}+q^{3 a+b-d-3 e} \tilde{E}_{\mathbf{2}}^{a, b, c, d, e, f+1} \\
& +[2]_{1}[b]_{1} q^{3(a-c)} \tilde{E}_{\mathbf{2}}^{a, b-1, c, d+1, e, f}+[a]_{2} \tilde{E}_{\mathbf{2}}^{a-1, b+1, c, d, e, f}, \\
e_{2} \cdot \tilde{E}_{\mathbf{2}}^{a, b, c, d, e, f}= & \tilde{E}_{\mathbf{2}}^{a+1, b, c, d, e, f} .
\end{aligned}
$$

Proof. By induction, we have

$$
\begin{aligned}
& b_{6} b_{1}^{n}=q^{3 n} b_{1}^{n} b_{6}+[n]_{2} b_{1}^{n-1} b_{2}, \\
& b_{6} b_{2}^{n}=[3]_{1} q^{2-n}[n-1]_{1}[n]_{1} b_{2}^{n-2} b_{3}+q^{n} b_{2}^{n} b_{6}+[2]_{1}[n]_{1} b_{2}^{n-1} b_{4}, \\
& b_{4} b_{3}^{n}=q^{-3 n} b_{3}^{n} b_{4}, \quad b_{6} b_{3}^{n}=b_{3}^{n} b_{6}-\langle 1\rangle q^{2-3 n}[n]_{2} b_{3}^{n-1} b_{4}^{2}, \\
& b_{6} b_{4}^{n}=[3]_{1} q^{2-2 n}[n]_{1} b_{4}^{n-1} b_{5}+q^{-n} b_{4}^{n} b_{6}, \quad b_{6} b_{5}^{n}=q^{-3 n} b_{5}^{n} b_{6},
\end{aligned}
$$

and

$$
\begin{aligned}
b_{6}^{n} b_{1}= & q^{n-2}[n-1]_{1}[n]_{1} b_{4} b_{6}^{n-2}+q^{3 n} b_{1} b_{6}^{n} \\
& +q^{2(n-1)}[n]_{1} b_{2} b_{6}^{n-1}+[n-2]_{1}[n-1]_{1}[n]_{1} b_{5} b_{6}^{n-3}, \\
b_{5}^{n} b_{1}= & \langle 1\rangle^{2} q^{-3(n-1)}[n-1]_{2}[n]_{2}[3]_{1}^{-1} b_{4}^{3} b_{5}^{n-2}+q^{3 n} b_{1} b_{5}^{n} \\
& -q^{-3}\left(q^{4}+q^{2}-1\right)[n]_{2} b_{3} b_{5}^{n-1}-q^{-1}\langle 1\rangle[n]_{2} b_{2} b_{4} b_{5}^{n-1},
\end{aligned}
$$




$$
\begin{aligned}
b_{5}^{n} b_{2}= & b_{2} b_{5}^{n}-\langle 1\rangle q^{2-3 n}[n]_{2} b_{4}^{2} b_{5}^{n-1}, \quad b_{5}^{n} b_{4}=q^{-3 n} b_{4} b_{5}^{n}, \\
b_{4}^{n} b_{1}= & -\langle 3\rangle q^{6-3 n}[n-2]_{1}[n-1]_{1}[n]_{1} b_{3}^{2} b_{4}^{n-3}-\langle 1\rangle q^{-n}[n]_{1} b_{2}^{2} b_{4}^{n-1} \\
& -\langle 3\rangle q^{1-2 n}[n-1]_{1}[n]_{1} b_{2} b_{3} b_{4}^{n-2}+b_{1} b_{4}^{n}, \\
b_{4}^{n} b_{2}= & {[3]_{1} q^{2-2 n}[n]_{1} b_{3} b_{4}^{n-1}+q^{-n} b_{2} b_{4}^{n}, \quad b_{4}^{n} b_{3}=q^{-3 n} b_{3} b_{4}^{n}, } \\
b_{3}^{n} b_{1}= & q^{-3 n} b_{1} b_{3}^{n}+\langle 1\rangle^{2} q^{3-6 n}[n]_{2}[3]_{1}^{-1} b_{2}^{3} b_{3}^{n-1}, \quad b_{3}^{n} b_{2}=q^{-3 n} b_{2} b_{3}^{n}, \quad b_{2}^{n} b_{1}=q^{-3 n} b_{1} b_{2}^{n} .
\end{aligned}
$$

The lemma is a direct consequence of these formulae.

A part of the above results have also been obtained in [25].

Let us turn to the representations $\pi_{\mathbf{i}}$ of $A_{q}\left(G_{2}\right)$. The elements $\sigma_{i}$ in Definition 1 and $\sigma_{i} e_{i}$ are given by

$$
\sigma_{1}=t_{17}, \quad \sigma_{2}=t_{26} t_{17}-q t_{27} t_{16}, \quad \sigma_{1} e_{1}=t_{27}, \quad \sigma_{2} e_{2}=t_{36} t_{17}-q t_{37} t_{16} .
$$

From (14) and (27) with $\alpha_{i}=\mu_{i}=1$, we have

$$
\begin{aligned}
& \pi_{\mathbf{1}}\left(\sigma_{1}\right)=\mathbf{k}_{1} \mathbf{K}_{2} \mathbf{k}_{3}^{2} \mathbf{K}_{4} \mathbf{k}_{5}, \\
& \pi_{\mathbf{1}}\left(\sigma_{2}\right)=\mathbf{K}_{2} \mathbf{k}_{3}^{3} \mathbf{K}_{4}^{2} \mathbf{k}_{5}^{3} \mathbf{K}_{6}, \\
& \pi_{\mathbf{1}}\left(\sigma_{1} e_{1}\right)=\mathbf{a}_{1}^{+} \mathbf{K}_{2} \mathbf{k}_{3}^{2} \mathbf{K}_{4} \mathbf{k}_{5} \text {, } \\
& \pi_{\mathbf{1}}\left(\sigma_{2} e_{2}\right)=\mathbf{k}_{1}^{3} \mathbf{K}_{2}^{2} \mathbf{k}_{3}^{3} \mathbf{K}_{4} \mathbf{A}_{6}^{+}+[2]_{2} \mathbf{k}_{1}^{3} \mathbf{A}_{2}^{-} \mathbf{K}_{2} \mathbf{A}_{4}^{+} \mathbf{K}_{4} \mathbf{k}_{5}^{3} \mathbf{K}_{6}+\mathbf{a}^{-3}{ }_{1} \mathbf{A}_{2}^{+} \mathbf{k}_{3}^{3} \mathbf{K}_{4}^{2} \mathbf{k}_{5}^{3} \mathbf{K}_{6} \\
& +[3]_{1} \mathbf{a}_{1}^{-2} \mathbf{k}_{1} \mathbf{a}_{3}^{+} \mathbf{k}_{3}^{2} \mathbf{K}_{4}^{2} \mathbf{k}_{5}^{3} \mathbf{K}_{6}+[3]_{1} \mathbf{a}_{1}^{-} \mathbf{k}_{1}^{2} \mathbf{K}_{2} \mathbf{k}_{3}^{2} \mathbf{K}_{4} \mathbf{a}_{5}^{+} \mathbf{k}_{5}^{2} \mathbf{K}_{6} \\
& -q[3]_{1} \mathbf{k}_{1}^{3} \mathbf{A}_{2}^{-} \mathbf{K}_{2} \mathbf{k}_{3}^{2} \mathbf{A}_{4}^{+} \mathbf{K}_{4} \mathbf{k}_{5}^{3} \mathbf{K}_{6}+[3]_{1} \mathbf{k}_{1}^{3} \mathbf{K}_{2}^{2} \mathbf{a}_{3}^{-} \mathbf{k}_{3}^{2} \mathbf{a}_{5}^{+2} \mathbf{k}_{5} \mathbf{K}_{6}+\mathbf{k}_{1}^{3} \mathbf{K}_{2}^{2} \mathbf{k}_{3}^{3} \mathbf{A}_{4}^{-} \mathbf{a}_{5}^{+3} \mathbf{K}_{6} \\
& +[3]_{1} \mathbf{a}_{1}^{-} \mathbf{k}_{1}^{2} \mathbf{A}_{2}^{-} \mathbf{a}_{3}^{+2} \mathbf{k}_{3} \mathbf{K}_{4}^{2} \mathbf{k}_{5}^{3} \mathbf{K}_{6}+[3]_{1} \mathbf{a}_{1}^{-} \mathbf{k}_{1}^{2} \mathbf{K}_{2} \mathbf{a}_{3}^{-} \mathbf{k}_{3} \mathbf{A}_{4}^{+} \mathbf{K}_{4} \mathbf{k}_{5}^{3} \mathbf{K}_{6} \\
& +\mathbf{k}_{1}^{3} \mathbf{A}_{2}^{-2} \mathbf{a}_{3}^{+3} \mathbf{K}_{4}^{2} \mathbf{k}_{5}^{3} \mathbf{K}_{6}+[3]_{1} \mathbf{k}_{1}^{3} \mathbf{A}_{2}^{-} \mathbf{K}_{2} \mathbf{a}_{3}^{+} \mathbf{k}_{3} \mathbf{K}_{4} \mathbf{a}_{5}^{+} \mathbf{k}_{5}^{2} \mathbf{K}_{6} \\
& +\mathbf{k}_{1}^{3} \mathbf{K}_{2}^{2} \mathbf{a}_{3}^{-3} \mathbf{A}_{4}^{+2} \mathbf{k}_{5}^{3} \mathbf{K}_{6}+[3]_{1} \mathbf{k}_{1}^{3} \mathbf{K}_{2}^{2} \mathbf{a}_{3}^{-2} \mathbf{k}_{3} \mathbf{A}_{4}^{+} \mathbf{a}_{5}^{+} \mathbf{k}_{5}^{2} \mathbf{K}_{6}, \\
& \lambda_{1}^{-1} \pi_{\mathbf{1}}\left(\xi_{1}\right)=\mathbf{a}_{1}^{+} \mathbf{k}_{1}^{-1}, \\
& \lambda_{2}^{-1} \pi_{\mathbf{1}}\left(\xi_{2}\right)=\mathbf{a}_{1}^{-3} \mathbf{A}_{2}^{+} \mathbf{K}_{2}^{-1}+[2]_{2} \mathbf{k}_{1}^{3} \mathbf{A}_{2}^{-} \mathbf{k}_{3}^{-3} \mathbf{A}_{4}^{+} \mathbf{K}_{4}^{-1}-q[3]_{1} \mathbf{k}_{1}^{3} \mathbf{A}_{2}^{-} \mathbf{k}_{3}^{-1} \mathbf{A}_{4}^{+} \mathbf{K}_{4}^{-1} \\
& +[3]_{1} \mathbf{a}_{1}^{-2} \mathbf{k}_{1} \mathbf{K}_{2}^{-1} \mathbf{a}_{3}^{+} \mathbf{k}_{3}^{-1}+[3]_{1} \mathbf{a}_{1}^{-} \mathbf{k}_{1}^{2} \mathbf{a}_{3}^{-} \mathbf{k}_{3}^{-2} \mathbf{A}_{4}^{+} \mathbf{K}_{4}^{-1}+[3]_{1} \mathbf{a}_{1}^{-} \mathbf{k}_{1}^{2} \mathbf{k}_{3}^{-1} \mathbf{K}_{4}^{-1} \mathbf{a}_{5}^{+} \mathbf{k}_{5}^{-1} \\
& +\mathbf{k}_{1}^{3} \mathbf{K}_{2} \mathbf{K}_{4}^{-1} \mathbf{k}_{5}^{-3} \mathbf{A}_{6}^{+} \mathbf{K}_{6}^{-1}+[3]_{1} \mathbf{a}_{1}^{-} \mathbf{k}_{1}^{2} \mathbf{A}_{2}^{-} \mathbf{K}_{2}^{-1} \mathbf{a}_{3}^{+2} \mathbf{k}_{3}^{-2} \\
& +[3]_{1} \mathbf{k}_{1}^{3} \mathbf{A}_{2}^{-} \mathbf{a}_{3}^{+} \mathbf{k}_{3}^{-2} \mathbf{K}_{4}^{-1} \mathbf{a}_{5}^{+} \mathbf{k}_{5}^{-1}+\mathbf{k}_{1}^{3} \mathbf{A}_{2}^{-2} \mathbf{K}_{2}^{-1} \mathbf{a}_{3}^{+3} \mathbf{k}_{3}^{-3} \\
& +[3]_{1} \mathbf{k}_{1}^{3} \mathbf{K}_{2} \mathbf{a}_{3}^{-} \mathbf{k}_{3}^{-1} \mathbf{K}_{4}^{-2} \mathbf{a}_{5}^{+2} \mathbf{k}_{5}^{-2}+\mathbf{k}_{1}^{3} \mathbf{K}_{2} \mathbf{A}_{4}^{-} \mathbf{K}_{4}^{-2} \mathbf{a}_{5}^{+3} \mathbf{k}_{5}^{-3} \\
& +\mathbf{k}_{1}^{3} \mathbf{K}_{2} \mathbf{a}_{3}^{-3} \mathbf{k}_{3}^{-3} \mathbf{A}_{4}^{+2} \mathbf{K}_{4}^{-2}+[3]_{1} \mathbf{k}_{1}^{3} \mathbf{K}_{2} \mathbf{a}_{3}^{-2} \mathbf{k}_{3}^{-2} \mathbf{A}_{4}^{+} \mathbf{K}_{4}^{-2} \mathbf{a}_{5}^{+} \mathbf{k}_{5}^{-1} \\
& \pi_{\mathbf{2}}\left(\sigma_{1}\right)=\mathbf{k}_{2} \mathbf{K}_{3} \mathbf{k}_{4}^{2} \mathbf{K}_{5} \mathbf{k}_{6}, \\
& \pi_{\mathbf{2}}\left(\sigma_{2}\right)=\mathbf{K}_{1} \mathbf{k}_{2}^{3} \mathbf{K}_{3}^{2} \mathbf{k}_{4}^{3} \mathbf{K}_{5} \text {, } \\
& \pi_{\mathbf{2}}\left(\sigma_{1} e_{1}\right)=\mathbf{K}_{1} \mathbf{k}_{2}^{2} \mathbf{K}_{3} \mathbf{k}_{4} \mathbf{a}_{6}^{+}+\mathbf{A}_{1}^{-} \mathbf{a}_{2}^{+} \mathbf{K}_{3} \mathbf{k}_{4}^{2} \mathbf{K}_{5} \mathbf{k}_{6}+\mathbf{K}_{1} \mathbf{k}_{2}^{2} \mathbf{K}_{3} \mathbf{a}_{4}^{-} \mathbf{A}_{5}^{+} \mathbf{k}_{6}+\mathbf{K}_{1} \mathbf{a}_{2}^{-2} \mathbf{A}_{3}^{+} \mathbf{k}_{4}^{2} \mathbf{K}_{5} \mathbf{k}_{6} \\
& +[2]_{1} \mathbf{K}_{1} \mathbf{a}_{2}^{-} \mathbf{k}_{2} \mathbf{a}_{4}^{+} \mathbf{k}_{4} \mathbf{K}_{5} \mathbf{k}_{6}+\mathbf{K}_{1} \mathbf{k}_{2}^{2} \mathbf{A}_{3}^{-} \mathbf{a}_{4}^{+2} \mathbf{K}_{5} \mathbf{k}_{6}, \\
& \pi_{\mathbf{2}}\left(\sigma_{2} e_{2}\right)=\mathbf{A}_{1}^{+} \mathbf{k}_{2}^{3} \mathbf{K}_{3}^{2} \mathbf{k}_{4}^{3} \mathbf{K}_{5} \\
& \lambda_{1}^{-1} \pi_{\mathbf{2}}\left(\xi_{1}\right)=\mathbf{A}_{1}^{-} \mathbf{a}_{2}^{+} \mathbf{k}_{2}^{-1}+[2]_{1} \mathbf{K}_{1} \mathbf{a}_{2}^{-} \mathbf{K}_{3}^{-1} \mathbf{a}_{4}^{+} \mathbf{k}_{4}^{-1}+\mathbf{K}_{1} \mathbf{a}_{2}^{-2} \mathbf{k}_{2}^{-1} \mathbf{A}_{3}^{+} \mathbf{K}_{3}^{-1}+\mathbf{K}_{1} \mathbf{k}_{2} \mathbf{a}_{4}^{-} \mathbf{k}_{4}^{-2} \mathbf{A}_{5}^{+} \mathbf{K}_{5}^{-1} \\
& +\mathbf{K}_{1} \mathbf{k}_{2} \mathbf{k}_{4}^{-1} \mathbf{K}_{5}^{-1} \mathbf{a}_{6}^{+} \mathbf{k}_{6}^{-1}+\mathbf{K}_{1} \mathbf{k}_{2} \mathbf{A}_{3}^{-} \mathbf{K}_{3}^{-1} \mathbf{a}_{4}^{+2} \mathbf{k}_{4}^{-2} \\
& \lambda_{2}^{-1} \pi_{\mathbf{2}}\left(\xi_{2}\right)=\mathbf{A}_{1}^{+} \mathbf{K}_{1}^{-1} .
\end{aligned}
$$

We find that $\pi_{\mathbf{i}}\left(\sigma_{i}\right)$ is invertible. Comparing these formulae with Lemma 4 by using (40), the equality (37) is directly checked. Thus Proposition 2 is established for $G_{2}$. 
In terms of the checked intertwiner $\mathcal{F}$ in Section 4.4, Theorem 5 implies

$$
E_{\mathbf{2}}^{a, b, c, d, e, f}=\sum_{i, j, k, l, m, n} \mathcal{F}_{i j k l m n}^{a b c d e f} E_{\mathbf{1}}^{n, m, l, k, j, i}
$$

\section{Discussion}

In view of Proposition 2 it is natural to expect that the map defined on generators of $U_{q}^{+}(\mathfrak{g})$ as $e_{i} \mapsto \eta_{i}:=\sigma_{i} e_{i} / \sigma_{i}$ extends to an algebra homomorphism from $U_{q}^{+}(\mathfrak{g})$ to $A_{q}(\mathfrak{g})_{\mathcal{S}}$, namely, $\eta_{i}$ satisfies $q$-Serre relations. In fact, it is true not only for rank 2 cases but also for any $\mathfrak{g}$.

Theorem 6. In $A_{q}(\mathfrak{g})_{\mathcal{S}}$ the following relation holds for any $i, j(i \neq j)$ :

$$
\sum_{r=0}^{1-a_{i j}}(-1)^{r} \eta_{i}^{(r)} \eta_{j} \eta_{i}^{\left(1-a_{i j}-r\right)}=0
$$

Proof. By relabeling of Dynkin indices we can assume $i=1, j=2$. Set $\tau_{i}=\sigma_{i} e_{i}$ for $i=1,2$. Then from Proposition 1 we have

$$
\sigma_{i} \tau_{i}=q_{i} \tau_{i} \sigma_{i}, \quad i=1,2, \quad \sigma_{i} \tau_{j}=\tau_{j} \sigma_{i}, \quad i, j=1,2 ; \quad i \neq j .
$$

Using (12) with these relations one verifies

$$
\eta_{1}^{r} \eta_{2} \eta_{1}^{s}=q_{1}^{(r+s)(r+s-1) / 2}\left(\tau_{1}^{r} \tau_{2} \tau_{1}^{s}\right) /\left(\sigma_{1}^{r} \sigma_{2} \sigma_{1}^{s}\right) .
$$

Here we have set $s=1-a_{12}-r$. Recalling that $\sigma_{1}$ and $\sigma_{2}$ commute with each other, we can reduce the claim to showing

$$
Z:=\sum_{r=0}^{1-a_{12}}(-1)^{r} \tau_{1}^{(r)} \tau_{2} \tau_{1}^{(s)}=0 .
$$

Note that the right (resp. left) weight of $Z$ is $\left(1-a_{12}\right)\left(\varpi_{1}-\alpha_{1}\right)+\left(\varpi_{2}-\alpha_{2}\right)$ (resp. $w_{0}((1-$ $\left.\left.\left.a_{12}\right) \varpi_{1}+\varpi_{2}\right)\right)$. The two weights are not related by the longest element $w_{0} \in W$. Hence if we show $f_{i} Z=Z f_{i}=0$ for any $i$, we can conclude $Z=0$ by the remark after Lemma 1 . The properties $f_{i} Z=0$ for any $i$ and $Z f_{i}=0$ for $i \neq 1,2$ are trivial.

First we show $Z f_{2}=0$. We have

$$
\left(\tau_{1}^{r} \tau_{2} \tau_{1}^{s}\right) f_{2}=\tau_{1}^{r}\left(\tau_{2} f_{2}\right)\left(\tau_{1} k_{2}^{-1}\right)^{s}=\tau_{1}^{r} \sigma_{2}\left(\beta \tau_{1}\right)^{s}=\beta^{s} \tau_{1}^{r+s} \sigma_{2}
$$

where $\beta=q_{2}^{-\left\langle h_{2}, \varpi_{1}-\alpha_{1}\right\rangle}=q_{2}^{a_{21}}=q_{1}^{a_{12}}$ and we have used (43). Hence,

$$
Z f_{2}=\left(\sum_{r+s=1-a_{12}} \frac{\left(-q_{1}^{-a_{12}}\right)^{s}}{[r]_{1} ![s]_{1} !}\right)\left(-\tau_{1}\right)^{1-a_{12}} \sigma_{2}=0
$$

In the last equality we have used the following formula:

$$
\sum_{i=0}^{m}(-z)^{i}\left[\begin{array}{c}
m \\
i
\end{array}\right]=\prod_{j=1}^{m}\left(1-z q^{2 j-m-1}\right)
$$

where $\left[\begin{array}{c}m \\ i\end{array}\right]=[m] ! /([i] ![m-i] !)$. 
Next, we show $Z f_{1}=0$.

$$
\begin{aligned}
\left(\tau_{1}^{r} \tau_{2} \tau_{1}^{s}\right) f_{1} & =\sum_{i=1}^{r} \tau_{1}^{r-i} \sigma_{1}\left(\tau_{1} k_{1}^{-1}\right)^{i-1}\left(\tau_{2} k_{1}^{-1}\right)\left(\tau_{1} k_{1}^{-1}\right)^{s}+\tau_{1}^{r} \tau_{2} \sum_{i=1}^{s} \tau_{1}^{s-i} \sigma_{1}\left(\tau_{1} k_{1}^{-1}\right)^{i-1} \\
& =\sum_{i=1}^{r} \delta \gamma^{i-1-s} \tau_{1}^{r-1} \tau_{2} \tau_{1}^{s} \sigma_{1}+\sum_{i=1}^{s} \gamma^{i-1} \tau_{1}^{r} \tau_{2} \tau_{1}^{s-1} \sigma_{1}
\end{aligned}
$$

where constants $\gamma, \delta$ are determined by $\sigma_{1}\left(\tau_{1} k_{1}^{-1}\right)=\gamma \tau_{1} \sigma_{1}, \sigma_{1}\left(\tau_{2} k_{1}^{-1}\right)=\delta \tau_{2} \sigma_{1}$ and hence we have $\gamma=q_{1} q_{1}^{-\left\langle h_{1}, \varpi_{1}-\alpha_{1}\right\rangle}=q_{1}^{2}, \delta=q_{1}^{-\left\langle h_{1}, \varpi_{2}-\alpha_{2}\right\rangle}=q_{1}^{a_{12}}$. Then, we obtain

$$
\begin{aligned}
Z f_{1} & =\sum_{r+s=1-a_{12}} \frac{(-1)^{r}}{[r]_{1} ![s]_{1} !}\left(\sum_{i=1}^{r} \delta \gamma^{i-1+s} \tau_{1}^{r-1} \tau_{2} \tau_{1}^{s} \sigma_{1}+\sum_{i=1}^{s} \gamma^{i-1} \tau_{1}^{r} \tau_{2} \tau_{1}^{s-1} \sigma_{1}\right) \\
& =\sum_{r+s=1-a_{12}} \frac{(-1)^{r}}{[r]_{1} ![s]_{1} !}\left(\delta \gamma^{s} \frac{1-\gamma^{r}}{1-\gamma} \tau_{1}^{r-1} \tau_{2} \tau_{1}^{s} \sigma_{1}+\frac{1-\gamma^{s}}{1-\gamma} \tau_{1}^{r} \tau_{2} \tau_{1}^{s-1} \sigma_{1}\right) \\
& =\sum_{r+s=1-a_{12}}\left(-(-1)^{r-1} q_{1}^{s} \tau_{1}^{(r-1)} \tau_{2} \tau_{1}^{(s)}+(-1)^{r} q_{1}^{s-1} \tau_{1}^{(r)} \tau_{2} \tau_{1}^{(s-1)}\right) \sigma_{1}=0
\end{aligned}
$$

as desired.

Remark 1. The special case $w=w_{0}$ of [26, Theorem 3.7] gives Theorem 6 here. Moreover $\left[26\right.$, Theorem 3.7] also shows that $U_{q}^{+}(\mathfrak{g})$ is isomorphic to an explicit subalgebra of $A_{q}(\mathfrak{g}) \mathcal{S}$. We would like to thank the referee for pointing this out and for giving helpful comments.

It will be interesting to investigate it further in the light of the quantum cluster algebra which has been recognized as a fundamental structure in the quantized algebra of functions [6]. The representations via multiplication on PBW bases also play a fundamental role in the study of the positive principal series representations and modular double [7].

In this paper we have not discussed the analogue of the tetrahedron and 3D reflection equations for general $\mathfrak{g}$. However, from our proof of Theorem 5 , we expect that the basic constituents are $R$ and $K$ only, and their compatibility condition is reduced to the rank 2 cases (2) and (3).

\section{Acknowledgments}

The authors thank Ivan C.H. Ip, Anatol N. Kirillov, Toshiki Nakashima and Masatoshi Noumi for communications. They also thank one of the referees for drawing attention to the references $[9,26]$. This work is supported by Grants-in-Aid for Scientific Research No. 23340007, No. 24540203, No. 23654007 and No. 21340036 from JSPS.

\section{References}

[1] Bazhanov V.V., Mangazeev V.V., Sergeev S.M., Quantum geometry of 3-dimensional lattices, J. Stat. Mech. Theory Exp. 2008 (2008), P07004, 27 pages, arXiv:0801.0129.

[2] Bazhanov V.V., Sergeev S.M., Zamolodchikov's tetrahedron equation and hidden structure of quantum groups, J. Phys. A: Math. Gen. 39 (2006), 3295-3310, hep-th/0509181.

[3] Berenstein A., Zelevinsky A., Total positivity in Schubert varieties, Comment. Math. Helv. 72 (1997), 128166.

[4] Chari V., Pressley A., A guide to quantum groups, Cambridge University Press, Cambridge, 1994.

[5] Drinfeld V.G., Quantum groups, in Proceedings of the International Congress of Mathematicians, Vols. 1, 2 (Berkeley, Calif., 1986), Amer. Math. Soc., Providence, RI, 1987, 798-820. 
[6] Geiß C., Leclerc B., Schröer J., Cluster structures on quantum coordinate rings, Selecta Math. (N.S.) 19 (2013), 337-397, arXiv:1104.0531.

[7] Ip I.C.H., Positive representations of split real quantum groups of type $B_{n}, C_{n}, F_{4}$, and $G_{2}$, arXiv:1205.2940.

[8] Isaev A.P., Kulish P.P., Tetrahedron reflection equations, Modern Phys. Lett. A 12 (1997), 427-437, hep-th/9702013.

[9] Joseph A., Quantum groups and their primitive ideals, Ergebnisse der Mathematik und ihrer Grenzgebiete (3), Vol. 29, Springer-Verlag, Berlin, 1995.

[10] Kapranov M.M., Voevodsky V.A., 2-categories and Zamolodchikov tetrahedra equations, in Algebraic Groups and their Generalizations: Quantum and Infinite-Dimensional Methods (University Park, PA, 1991), Proc. Sympos. Pure Math., Vol. 56, Amer. Math. Soc., Providence, RI, 1994, 177-259.

[11] Kashiwara M., Global crystal bases of quantum groups, Duke Math. J. 69 (1993), 455-485.

[12] Kuniba A., Okado M., A solution of the 3D reflection equation from quantized algebra of functions of type $B$, arXiv: 1210.6430 .

[13] Kuniba A., Okado M., Tetrahedron and 3D reflection equations from quantized algebra of functions, J. Phys. A: Math. Theor. 45 (2012), 465206, 27 pages, arXiv:1208.1586.

[14] Lusztig G., Canonical bases arising from quantized enveloping algebras, J. Amer. Math. Soc. 3 (1990), 447-498.

[15] Lusztig G., Introduction to quantum groups, Progress in Mathematics, Vol. 110, Birkhäuser Boston Inc., Boston, MA, 1993.

[16] McConnell J.C., Robson J.C., Noncommutative Noetherian rings, Pure and Applied Mathematics (New York), John Wiley \& Sons Ltd., Chichester, 1987.

[17] Noumi M., Yamada H., Mimachi K., Finite-dimensional representations of the quantum group $\mathrm{GL}_{q}(n ; \mathbf{C})$ and the zonal spherical functions on $\mathrm{U}_{q}(n-1) \backslash \mathrm{U}_{q}(n)$, Japan. J. Math. 19 (1993), 31-80.

[18] Reshetikhin N.Yu., Takhtadzhyan L.A., Faddeev L.D., Quantization of Lie groups and Lie algebras, Leningrad Math. J. 1 (1990), 193-225.

[19] Sasaki N., Quantization of Lie group and algebra of $G_{2}$ type in the Faddeev-Reshetikhin-Takhtajan approach, J. Math. Phys. 36 (1995), 4476-4488.

[20] Sergeev S.M., Tetrahedron equations and nilpotent subalgebras of $\mathcal{U}_{q}\left(\mathrm{sl}_{n}\right)$, Lett. Math. Phys. 83 (2008), 231-235, arXiv:0707.4029.

[21] Soibelman Y.S., Algebra of functions on a compact quantum group and its representations, Leningrad Math. J. 2 (1991), 161-178.

[22] Soibelman Y.S., Selected topics in quantum groups, Internat. J. Modern Phys. A 7 (1992), suppl. 1B, 859-887.

[23] Vaksman L.L., Soibelman Y.S., An algebra of functions on the quantum group SU(2), Funct. Anal. Appl. 22 (1988), 170-181.

[24] Xi N.H., Canonical basis for type $B_{2}$, J. Algebra 214 (1999), 8-21.

[25] Xi N.H., On the PBW bases of the quantum group $U_{v}\left(G_{2}\right)$, Algebra Colloq. 2 (1995), 355-362.

[26] Yakimov M., Invariant prime ideals in quantizations of nilpotent Lie algebras, Proc. Lond. Math. Soc. (3) 101 (2010), 454-476, arXiv:0905.0852.

[27] Zamolodchikov A.B., Tetrahedra equations and integrable systems in three-dimensional space, Soviet Phys. JETP 52 (1980), 325-336. 